\title{
Characterization of Weathering Processes of the Giant Copper Deposit of Tizert (Igherm Inlier, Anti-Atlas, Morocco)
}

\author{
Julien Poot $^{1, *}$, Michèle Verhaert ${ }^{1}\left(\mathbb{D}\right.$, Augustin Dekoninck ${ }^{1}{ }^{\mathbb{D}}$, Abdellah Oummouch ${ }^{2}$, \\ Abdelaziz El Basbas ${ }^{3}$, Lhou Maacha ${ }^{2}$ and Johan Yans ${ }^{1}$ \\ 1 Department of Geology, Institute of Life-Earth-Environment (ILEE), Université of Namur, Rue de \\ Bruxelles 61, 5000 Namur, Belgium; michele-alexandra.verhaert@unamur.be (M.V.); \\ augustin.dekoninck@unamur.be (A.D.); johan.yans@unamur.be (J.Y.) \\ 2 Managem Group, BP 5199, Casablanca 20100, Morocco; A.OUMMOUCH@managemgroup.com (A.O.); \\ L.maacha@managemgroup.com (L.M.) \\ 3 Département des Sciences de la Terre, Ecole Nationale Supérieure des Mines de Rabat, Avenue Ahmed \\ Cherkaoui, BP 753, Agdal, Rabat 10000, Morocco; abdelazizelbasbas@gmail.com \\ * Correspondence: julien.poot@unamur.be
}

Received: 4 June 2020; Accepted: 8 July 2020; Published: 10 July 2020

\begin{abstract}
The giant Tizert copper deposit is considered as the largest copper resource in the western Anti-Atlas (Morocco). The site is characterized by $\mathrm{Cu}$ mineralization carried by malachite, chalcocite, covellite, bornite and chalcopyrite; azurite is not observed. The host rocks are mainly limestones (Formation of Tamjout Dolomite) and sandstones/siltstones (Basal Series) of the Ediacaran/Cambrian transition. The supergene enrichment is most likely related to episodes of uplift/doming (last event since $30 \mathrm{Ma}$ ), which triggered the exhumation of primary/hypogene mineralization (chalcopyrite, pyrite, galena, chalcocite I and bornite I), generating their oxidation and the precipitation of secondary/supergene sulfides, carbonates and Fe-oxyhydroxides. The Tizert supergene deposit mainly consists of (i) a residual patchwork of laterite rich in Fe-oxyhydroxides; (ii) a saprolite rich in malachite, or "green oxide zone" where primary structures such as stratification are preserved; (iii) a cementation zone containing secondary sulfides (covellite, chalcocite II and bornite II). The abundance of $\mathrm{Cu}$ carbonates results from the rapid neutralization of acidic meteoric fluids, due to oxidation of primary sulfides, by carbonate host rocks. Chlorite is also involved in the neutralization processes in the sandstones/siltstones of the Basal Series, in which supergene clays, such as kaolinite and smectites, subsequently precipitated. At Tizert, as can be highlighted in other supergene $\mathrm{Cu}$-deposits around the world, azurite is absent due to low $\mathrm{pCO}_{2}$ and relatively high $\mathrm{pH}$ conditions. In addition to copper, $\mathrm{Ag}$ enrichment is also observed in weathered rocks; Fe-oxyhydroxides contain high $\mathrm{Zn}, \mathrm{As}$, and $\mathrm{Pb}$ contents. However, these secondary enrichments are quite low compared to $\mathrm{Cu}$ in the whole Tizert site, which is therefore, considered as relatively homogeneous.
\end{abstract}

Keywords: Tizert deposit; copper; weathering; supergene; western Anti-Atlas; Morocco; malachite; azurite; Tamjout Dolomite; lower limestone; basal series

\section{Introduction}

Since the 2000s, the increasing request for metals and rare earth elements for new technologies initiated/restarted the interest in supergene deposits. The extraction for such deposits is easier and faster than for hypogene ores thanks to their near-surface situation and their metal content usually being higher than in the primary ore [1]. Moreover, recent advances in hydrometallurgical acid leaching, 
solvent extraction (SX) and electron-winning techniques [2,3] facilitate post-extraction treatments for non-sulfide ores (e.g., carbonates, such as malachite).

In this context, exploration of new supergene targets has been carried out in many countries, including Morocco, during the last decade. The Tizert site (western Anti-Atlas, Morocco) is considered as the largest copper deposit in the western Anti-Atlas [4]. This deposit was discovered in 1969. Using several mining galleries, the site was first studied between 1970 and 1975 by the Bureau des Recherches Pétrolières et Minières (BRPM), which estimated the reserves to be 1,062,000 $t$ with 2.3\% $\mathrm{Cu}$ and $65 \mathrm{~g} / \mathrm{t} \mathrm{Ag} \mathrm{[5].} \mathrm{Since} \mathrm{2011,} \mathrm{exploration} \mathrm{studies} \mathrm{have} \mathrm{been} \mathrm{carried} \mathrm{out} \mathrm{by} \mathrm{the} \mathrm{Managem} \mathrm{group;}$ the resources are now estimated to be $56,820,000 \mathrm{t}$ with $1.03 \% \mathrm{Cu}$ and $23 \mathrm{~g} / \mathrm{t} \mathrm{Ag}$ [4]. Other copper deposits are currently mined in this part of the Anti-Atlas, especially in Neoproterozoic to Early Cambrian rocks: the Tazalaght mine (reserves of $260,000 \mathrm{t}$ with $1.89 \% \mathrm{Cu}$ ) $[5,6]$, the Ouansimi mine (reserves of 1,087,000 $\mathrm{t}$ with $2.63 \% \mathrm{Cu}$ ) [7,8] and the Agoujgal mine (reserves of 5,000,000 $\mathrm{t}$ with $1 \% \mathrm{Cu}$ and $20 \mathrm{~g} / \mathrm{t} \mathrm{Ag})[6,9]$.

In their pioneering characterization of the Tizert deposit, the authors in reference [4] mainly (i) highlight mineralogical phases observed in this area, focusing on primary (hypogene) mineral phases; (ii) evaluate its potential for possible exploitation (grades and tonnage). In this study, we complete the primary mineralization data proposed by the authors in reference [4], by characterizing the supergene mineral assemblages in order to determine their formation processes and identify possible enrichment associated to weathering in the Tizert giant $\mathrm{Cu}$ deposit.

\subsection{Geodynamic Context}

The Anti-Atlas has been studied for decades and papers dealing with its complex geodynamic evolution are widespread in the literature e.g., references [10,11]. The Anti-Atlas is located at the northern edge of the West African Craton (WAC) in Morocco. It extends over nearly $800 \mathrm{~km}$, following an ENE-WSW direction, over $200 \mathrm{~km}$ wide [12]. It is separated from the High Atlas and Meseta domains to the south by the Tindouf Basin, and to the north, by the South Atlas Fault (SAF) and the Souss and Ouarzazate basins (Figure 1) [13]. The Anti-Atlas culminates at $3200 \mathrm{~m}$ at Jbel Siroua and reaches up to $1800 \mathrm{~m}$ in altitude in the plateaus [11,14].

Between Late Neoproterozoic and Early Cambrian, the Anti-Atlas has been affected by extension and rifting episodes related to the opening of the Iapetus Ocean. This resulted in the formation of graben and half-graben structures filled by coarse clastic sediments and alkaline basalts (Tata and Taroudant group), indicating an intracontinental extension $[15,16]$. The post-rifting stage, during the Cambrian, is characterized by a significant subsidence of the Anti-Atlas and accumulation of fine clastic sediments thicker in the west than in the east [17-19].

Uplift and exhumation-The uplift and exhumation of the Precambrian basement, as well as the erosion of the Paleozoic cover, are associated with three major tectonic events. (1) Between Late Carboniferous and Early Permian, the Variscan compression caused significant inversion and folding in the Paleozoic basins of the Anti-Atlas, with a NW-SE main direction of shortening $[10,14,17]$. This thick-skinned deformation affected the sedimentary cover and the basement, from the Meseta plateau in the north to Mauritanians in the southern part of the Anti-Atlas $[17,20]$. (2) Between Late Permian and Early Triassic, the Anti-Atlas was affected by vertical movements caused by the Tethys and Central Atlantic rifting [14,21,22]. These events confirmed that the Anti-Atlas behaved as a rift shoulder of the Central Atlantic opening [23]. In the Early Cretaceous, cooling of the bedrock continued and erosion locally reached Precambrian rocks [24]. During the Cenomanian-Turonian transgression period, the Anti-Atlas was covered by shallow water carbonate deposits that were subsequently nearly complete eroded between the Senonian and the Cenozoic $[19,25,26]$. (3) During the Cenozoic, a major uplift took place within the Atlas chains following the convergence of the African and Eurasian continents $[27,28]$. In addition, between the Upper Eocene and Pleistocene, the Anti-Atlas was affected by minor deformations, involving a hot spot moving along the Moroccan coast and affecting the chain with a thermal uplift around $30 \mathrm{Ma}[23,28,29]$. This large-scale uplift, which mainly affected the 
western part of the Anti-Atlas, is associated with an average erosion of $0.08 \mathrm{~km} / \mathrm{Ma}$ since $30 \mathrm{Ma}$ [30]. Overall, episodes of exhumation and subsidence coincide with major thermal and tectonic events in the Anti-Atlas [23].

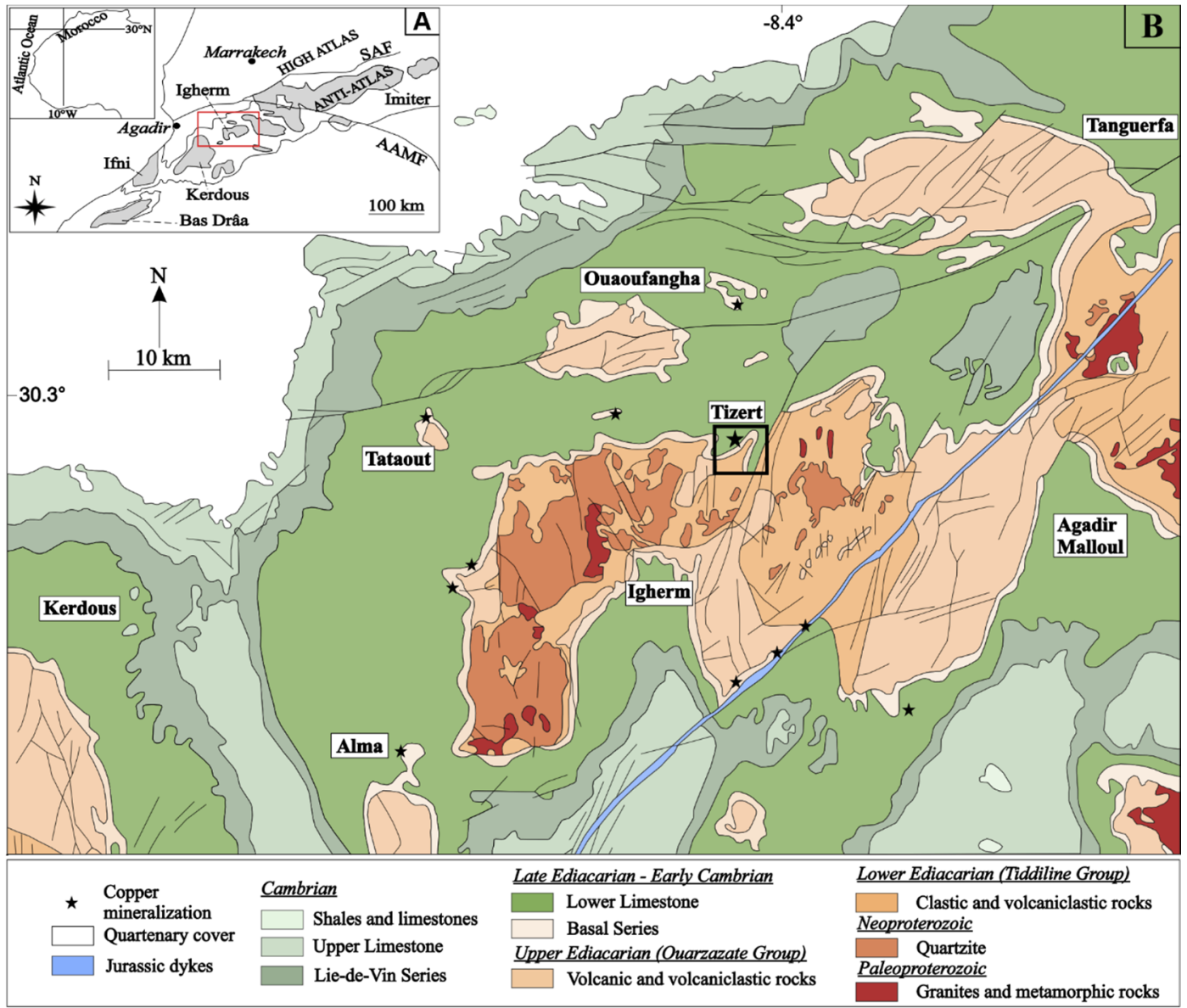

Figure 1. (A) General map of the Anti-Atlas; (B) Simplified geological map of the Igherm inlier (black square shows Tizert aera) (Modified after reference [4]).

Inliers-The Anti-Atlas is characterized by an alignment of inliers (Bas Drâa, Ifni, Kerdous, Igherm, Zenaga, Bou Azzer, J. Saghro and Ougnat; Figure 1B) along the highest axis and two major fault zones: Anti-Atlas Major Fault (AAMF) and South Atlas Fault (SAF). They expose the Precambrian metamorphic basement generally hidden under folded Paleozoic rocks [11,13]. During the Tonian-Cryogenian, sedimentary series composed of quartzites and limestones were deposited and later deformed and recrystallized during the Pan-African orogeny (686-660 Ma) [10]. To the south of the AAMF, the basement is mainly composed of shales and granites folded and metamorphosed during the Eburnean and Pan-African cycles e.g., references $[10,16]$. These rocks are overlaid by a Paleoproterozoic cover and Late Ediacaran volcanoclastic series, slightly deformed and unconformably disposed with the Eburnean and Pan-African series (Anezi and Ouarzazate/Tanalt Group) [14,31]. To the north of the AAMF (Figure 1A), the basement is mainly Neoproterozoic and consists of fragments of Cryogenian terrains composed of ophiolites and island arc gneisses that were located at the northern edge of the West African Craton during the paroxysm of the Pan-African orogeny [16,31,32]. As in the south, this domain is covered by the same series of Upper Ediacaran age [31]. All these series are unconformably overlaid by Paleozoic sediments up to $12 \mathrm{~km}$ thick in both sides of the Anti-Atlas Major Fault [10,16]. 


\subsection{Local Geologic Context}

The Tizert copper deposit is located at the northern edge of the Igherm inlier, which is composed of Paleoproterozoic to Lower Cambrian formations (Figures 1B and 2). Mineralization is mainly carried out by malachite $\left[\mathrm{Cu}_{2} \mathrm{CO}_{3}(\mathrm{OH})_{2}\right]$ and secondary sulfides such as chalcocite II $\left(\mathrm{Cu}_{2} \mathrm{~S}\right)$ or covellite $(\mathrm{CuS})$. According to reference [4], silver is represented by minerals such as stromeyerite (CuAgS), eugenite $\left(\mathrm{Ag}_{11} \mathrm{Hg}_{2}\right)$ or chlorargyrite $(\mathrm{AgCl})$.

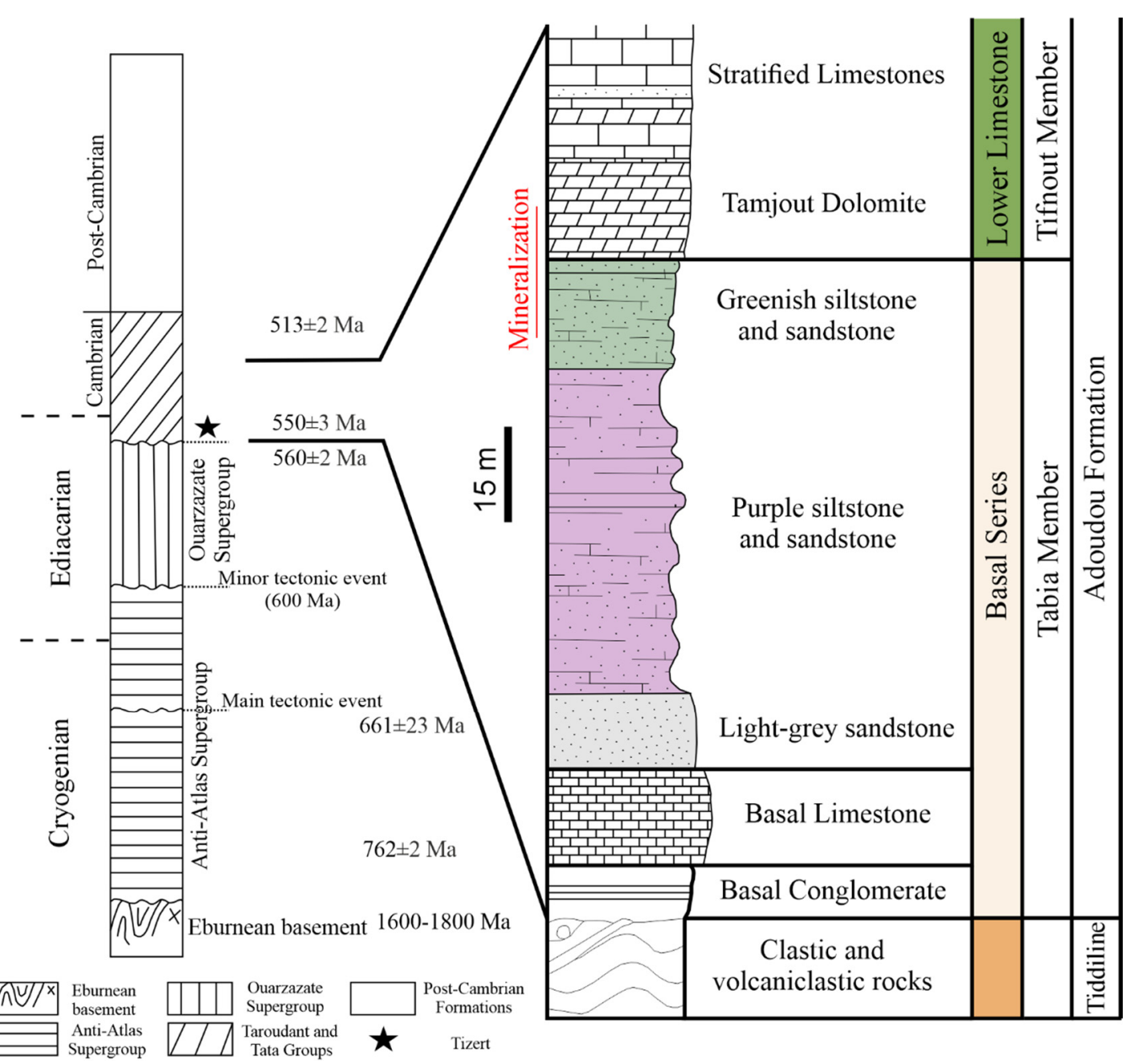

Figure 2. Simplified lithostratigraphy of the Anti-Atlas (left) and major geological units observed in the Tizert deposit (right) (Modified from references $[4,13,33]$ ).

The Proterozoic basement is mainly composed of metamorphic rocks (orthogneiss and migmatites) and granitic intrusions formed during the Eburnean orogeny. These granites, dated at $2050 \pm 6 \mathrm{Ma}$ (U-Pb on zircons), are porphyritic, mylonitized and generally weathered; their chemical composition is close to a subalkaline peraluminous adamellite (rich in Ba, Sr, Zr and Rare Earth Elements REE) [34]. The Tonian-Cryogenian limestones and quartzites (Lkest and Taghdout Group) have been deformed and recrystallized during the Pan-African orogeny [10]. In the Lower Ediacaran, late orogenic clastic and volcanoclastic series were deposited and deformed by a second event around 600 Ma [35,36]; this is the Tiddiline Formation (Figure 2), which constitutes the oldest rocks outcropping in the Tizert region. The second part of the Ediacaran is characterized by late to post-orogenic collapses, leading to an accumulation of nearly $2 \mathrm{~km}$ of conglomerates, volcanic and volcanoclastic rocks from the Ouarzazate Group (600-560 Ma), unconformably disposed with Eburnean and Pan-African series e.g., references [37,38]. This group, such as the Tiddiline series, is very small or absent in the Tizert area [4]. 
The Adoudou Formation (Figure 2) unconformably overlies the Ouarzazate Supergroup, with an angular unconformity of up to $20^{\circ}$ [37]. It was deposited between the Late Ediacaran and the Early Cambrian (between $550 \pm 3$ and $521 \pm 7 \mathrm{Ma}$, according to reference [13] and between $561 \pm 1$ and $524.84 \pm 0.09 \mathrm{Ma}$ according to reference [39]). The Adoudou Formation, forming the base of the Taroudant Group [39], was deposited during a major marine transgression from west to southeast; sedimentary units are thicker and carbonates more abundant in the western part [15]. It is generally divided into two members (Figure 2) — the Tabia and the Tifnout Members [40]. The Tabia Member corresponds to the "Basal Series" and is composed of different units, from base to top: (1) a massive Basal Conglomerate consisting of fluvial sandstones, coarse silico-clastic debris and silts; (2) peritidal carbonates, dolostones and silts; (3) sandstones and silts (grey, purple, greenish) [15,40,41]. The thickness of this "Basal Series" varies in the Tizert region from absent to $100 \mathrm{~m}$ thick [4].

The upper part of the Adoudou Formation is defined by the Tifnout Member. Its base consists of a 50-200 m thick dolostone, the Tamjout Dolomite, generally described as a partially silicified stromatolite dolostone formation [4,41]. Above this massive dolostone, the Tifnout Member is composed of parasequences (metric scale) composed of marls, dolostones and siltstones at the base, and also micritic and grainstone carbonates [39,40]. Trachytic and andesitic flows, dated at $534 \pm 10 \mathrm{Ma}$ on the Jbel Boho syenites, may also occur within the Tifnout Member [40]. The Tizert Cu mineralization is mainly located at the base of the Tamjout Dolomite and in the upper part of the Tifnout Member (Figure 2) [4]. Above the Adoudou Formation lies the "Lie-de-Vin" series, which are associated with a tilting in the Anti-Atlas margin [40]. They are composed of purplish silts/argillites formed by an increased supply of silico-clastic sediments to the basins, and of dolomitic limestones [4,41,42]. These series do not outcrop in the Tizert region.

\section{Materials and Methods}

Fifty-five samples were collected in March 2018, twenty-three from the surface (TIZ " X", e.g., TIZ 15) and others from three boreholes (C1, C15 and C75; Figure 3), in order to have the greatest diversity in terms of primary and secondary minerals. X-Ray Diffraction was carried out on whole rock samples in order to identify the major mineral phases. XRD analyses of powdered samples were performed using an X-Ray Panalytical X'Pert Pro diffractometer and a PHILLIPS PW3710 (CuK $\alpha$ radiation) at the PC2 platform (UNamur), operating at $40 \mathrm{kV}$ and $30 \mathrm{~mA}$. Nine samples containing clays were also analyzed by XRD. The isolation of the $<2 \mu \mathrm{m}$ size fraction and the analyses were performed following the methodology in reference [43].

Seventeen polished sections (mineralized samples) and fourteen thin sections were prepared at the Geology Department of the University of Namur (Namur, Belgium) and at the Laboratorio Petrografico (OMT di Canepa Pierluigi, Italy), respectively. They were studied using a petrographic polarizing microscope in transmitted and reflected light. A JEOL JSM 7500F scanning electron microscope (SEM), coupled with an energy dispersive spectrometer (EDS), was used to carry semi-quantitative analyses in order to identify some mineral phases at the Microscopy Service of the University of Namur.

Geochemical analyses were carried out on twenty-five representative samples (thirteen from surface and twelve from boreholes, Figure 4A-F) first analyzed by XRD, SEM or/and transmitted/reflected light microscopy. Crushing was performed with RETSCH PM 100 planetary ball mills (University of Namur) in order to obtain the maximum $125 \mu \mathrm{m}$ grain size. Agate crushing mortar was used for most samples, except for iron oxides, where a stainless-steel mortar was used to avoid contamination. The various analyses were carried out by Activation Laboratories Ltd. (Actlabs) (Ancaster, ON, Canada). Rare earth elements were analyzed by FUS-MS (Fusion Mass Spectrometry) and major elements by FUS-ICP (Fusion Inductively Coupled Plasma Optical Emission Spectrometry). The reduced iron (FeO) was determined by titration. Trace elements were also analyzed by FUS-MS; in some cases, $\mathrm{Cu}$ and $\mathrm{Pb}$ were quantified in percents rather than in ppm by FUS-Na2O2 (Fusion Inductively Coupled Plasma Sodium Peroxide Oxidation). Major, trace and rare earth elements were normalized to the composition of the upper continental crust (UCC, Upper Continental Crust) [44] to highlight geochemical trends. 
Host rocks, mineralized and weathered samples were compared to show possible enrichment or depletion for each element.

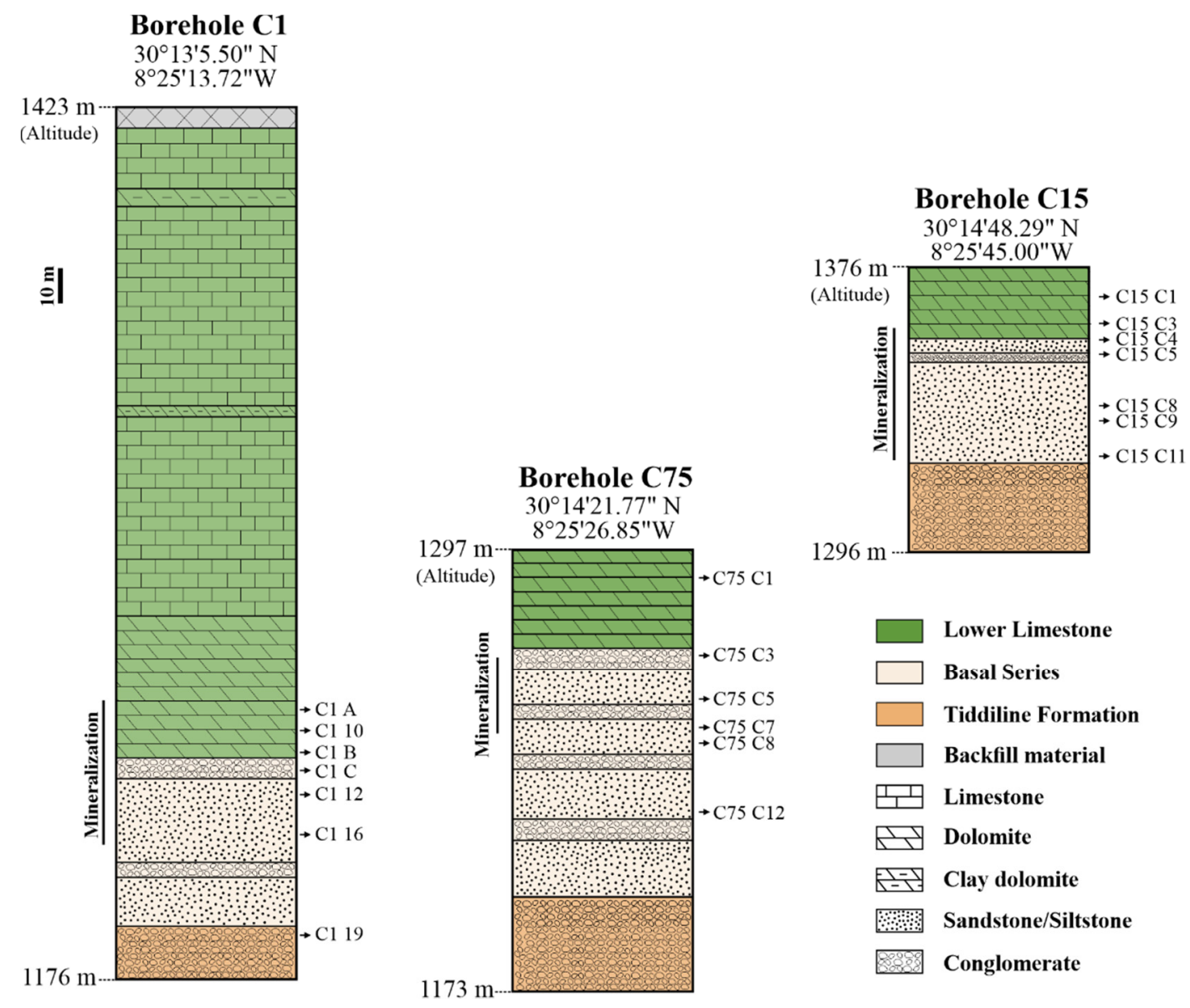

Figure 3. Schematic representation of studied boreholes $(\mathrm{C} 1, \mathrm{C} 15$ and $\mathrm{C} 75)$ with different analyzed samples.

\section{Results}

\subsection{Host Rocks}

The petrography, mineralogy and geochemistry of the host rocks play a crucial role in the formation of weathered ores [45]. In the Tizert area, most of host rocks (Tamjout Dolomite and the Basal Series-without the Basal Conglomerate) were collected at the Issedrin section, a few kilometers NW of the Tizert deposit.

Other host rock samples were collected from three boreholes of the Tizert site (C1, C15 and C75; Figure 3) in order to allow chemical comparison with weathered samples. In the boreholes, lithologies equivalent to those of the Issedrin section were analyzed: Tamjout Dolomite (C75 C1), red sandstone (C75 C3), purplish siltstone (C75 C8) and greenish siltstone (C75 C12). It is important to highlight that some of the host rocks, considered as "fresh", are slightly mineralized by a very low proportion of primary sulfides. Table 1 shows geochemical analyses and Figure 5A-E shows microphotographs for all these host rocks.

\subsubsection{Tamjout Dolomite}

This is a relatively pure dolostone made up of dolomite, calcite, quartz and muscovite. Calcite and quartz are mainly observed in nodules and veins. The presence of galena in one Tamjout Dolomite sample of the Issedrin site is confirmed by geochemical analyses (e.g., TIZ 45, Table 1) and will be 
described in the primary mineralization section (see below). There are no other observations of primary sulfides in the Issedrin site. The grades of $\mathrm{Ag}(0.6 \mathrm{ppm}), \mathrm{As}(5 \mathrm{ppm})$ and $\mathrm{Cu}(30 \mathrm{ppm})$ for the borehole sample (C75 C1) are relatively high in comparison with the $\mathrm{UCC}$, while $\mathrm{Pb}$ (5 ppm) is quite depleted (Table 1), although no primary sulfide was observed in this sample. The Tamjout Dolomite (surface and borehole, Figure $4 \mathrm{~A}$ ) is depleted in REE compared to the UCC. The sample (C75 C1) from the Tizert borehole and the TIZ 45 sample from the Issedrin section are, thus, weakly mineralized. All the other samples from the boreholes and from the Issedrin section are considered as "fresh" (= not mineralized).

\subsubsection{Basal Series}

The Basal Series contains sandstones, siltstones and conglomerates showing various colours (light-grey, purplish, greenish and red) and limestone ("Petit Calcaire"). The upper part is characterized by a light-grey sandstone (TIZ 44) made of quartz, muscovite, chlorite (Figure 5A) and feldspars (mostly orthoclase and albite). The carbonate content is quite low (Ca content of $0.4 \mathrm{wt} . \%$ and LOI 1.4 wt.\%). This rock, like most samples from the Basal Series, show an Ag content (1.1 ppm) similar to the one observed in the Tamjout Dolomite (Table 1). The REE profile is somewhat the same as the UCC, except for HREE (Heavy Rare Earth Elements), the latter being slightly depleted (Figure 4A).

Purplish and greenish siltstones are mostly observed in the upper and central part of the series. Purplish siltstones are made up of quartz, muscovite, chlorite, feldspars and hematite. Grain size is relatively small (max. $0.15 \mathrm{~mm}$ ) and the matrix is mainly composed of hematite and quartz, whereas the cement is slightly carbonated. $\mathrm{SiO}_{2}$ content (58.7 wt.\%) is lower than the light-grey sandstone (75.5 wt.\%), while $\mathrm{Fe}_{2} \mathrm{O}_{3}$ is higher (5.9 wt.\%), mostly due to hematite (responsible for the purple coloration) and chlorite. The $\mathrm{Al}_{2} \mathrm{O}_{3}, \mathrm{~K}_{2} \mathrm{O}$ and $\mathrm{TiO}_{2}$ contents are the highest among host rocks (Table 1 ). The REE pattern shows slight but not significative enrichment for surface samples, which is not observed in the boreholes (Figure 4A).

For trace elements, $\mathrm{Ag}$ (1.5 to $4.2 \mathrm{ppm}$ for the drilling sample) and Sb enrichment are well marked (Figure 4C). The greenish siltstone (TIZ 43) is made up of quartz, chlorite, muscovite, dolomite and feldspars. Like in the purplish siltstone, grain size does not exceed $0.15 \mathrm{~mm}$ and the cement is slightly carbonated. The proportion of chlorite is much higher than in other rocks of the Issedrin section and is the origin of the greenish coloration. $\mathrm{Cu}$ content is very low ( $<10 \mathrm{ppm}), \mathrm{SiO}_{2}$ content is quite similar to the purplish siltstone (Table 1), Ag and Sb grades are well marked (Figure 4C) and REE pattern (Figure 4A) shows a slight enrichment compared to the UCC. The composition of major elements in the greenish siltstone from the borehole (e.g., C75 C12) is quite different, mainly due to a lower proportion of chlorite, a higher proportion of quartz and the presence of microconglomerate clasts.

In the central part of the Basal Series, red sandstone (e.g., TIZ 41, Figure 5B) is composed of dolomite, quartz, muscovite, chlorite and orthoclase. Grain size can reach $2 \mathrm{~mm}$ for quartz, while chlorite and orthoclase do not exceed $0.5 \mathrm{~mm}$. The proportion of carbonates (cement and matrix) is higher than in other sandstones and siltstones of the Basal Series. The enrichments in As, Ag and Sn are significant (Figure 4C, respectively, 45, 26 and 15 times the UCC values). In addition, a slight enrichment in $\mathrm{Cu}$ can be observed, such as in the Tamjout Dolomite. The composition of this red sandstone is variable, especially regarding the proportion of chlorite and carbonates (e.g., TIZ 40, lower proportions), which are generally balanced by $\mathrm{SiO}_{2}$ (quartz) content. 
Table 1. Results of geochemical analyses for selected samples of host rocks and mineralization/weathered rocks. Major elements are listed in \%, while minor elements and REE are listed in ppm, except for values with an asterisk $\left(^{*}\right)$ which are in \%. LOD—Limit of Determination.

\begin{tabular}{|c|c|c|c|c|c|c|c|c|c|c|c|c|c|c|c|c|c|c|c|c|c|c|c|c|c|c|}
\hline \multirow{2}{*}{\multicolumn{2}{|c|}{ Oxides/Elements }} & \multicolumn{11}{|c|}{ Host Rocks } & \multicolumn{14}{|c|}{ Mineralization } \\
\hline & & \multirow[t]{2}{*}{ 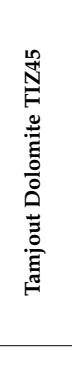 } & \multirow[t]{2}{*}{ 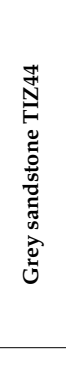 } & \multirow[t]{2}{*}{ 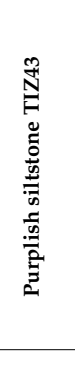 } & \multirow[t]{2}{*}{ 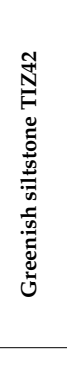 } & \multirow[t]{2}{*}{ 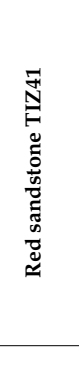 } & \multirow[t]{2}{*}{ 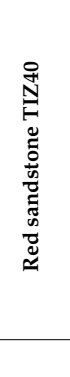 } & \multirow[t]{2}{*}{ 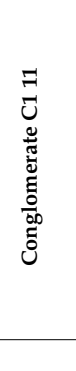 } & \multirow[t]{2}{*}{ 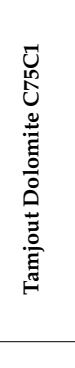 } & \multirow[t]{2}{*}{ 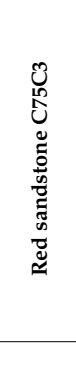 } & \multirow[t]{2}{*}{ 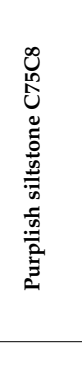 } & \multirow[t]{2}{*}{ 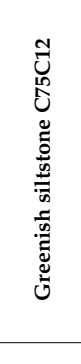 } & \multirow[t]{2}{*}{ 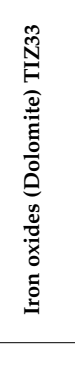 } & \multirow[t]{2}{*}{ 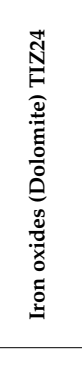 } & \multirow[t]{2}{*}{ 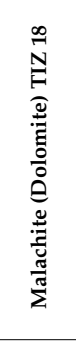 } & \multirow[t]{2}{*}{ 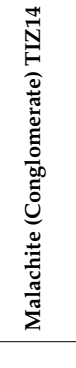 } & \multirow[t]{2}{*}{ 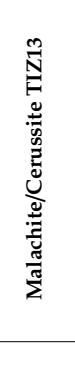 } & \multirow[t]{2}{*}{ 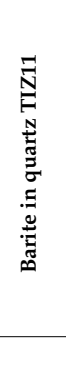 } & \multirow[t]{2}{*}{ 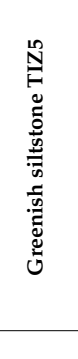 } & \multirow[t]{2}{*}{ 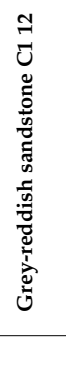 } & \multirow{3}{*}{ 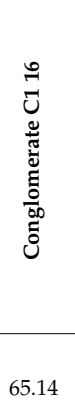 } & \multirow[t]{3}{*}{ 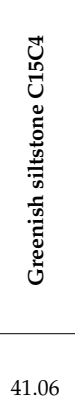 } & 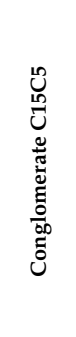 & 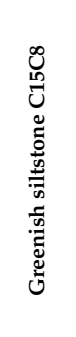 & 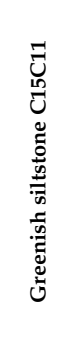 & 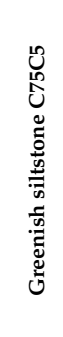 \\
\hline & LOD & & & & & & & & & & & & & & & & & & & & & & & & & \\
\hline $\mathrm{SiO}_{2}(\%)$ & 0.01 & 0.91 & 75.55 & 62.86 & 62.86 & 61.15 & 78.87 & 61.52 & 0.61 & 45.04 & 65.05 & 65.05 & 5.81 & 7.98 & 1.00 & 59.24 & 6.40 & 37.69 & 66.58 & 47.56 & & & 55.94 & 59.49 & 56.15 & 56.98 \\
\hline $\mathrm{Al}_{2} \mathrm{O}_{3}(\%)$ & 0.01 & 0.31 & 11.25 & 16.79 & 16.79 & 12.01 & 10.73 & 13.91 & 0.14 & 9.57 & 15.31 & 15.31 & 0.62 & 1.29 & 0.08 & 13.16 & 0.02 & 1.06 & 14.40 & 11.10 & 12.11 & 4.82 & 9.16 & 13.00 & 10.09 & 18.04 \\
\hline $\mathrm{Fe}_{2} \mathrm{O}_{3}(\%)$ & 0.01 & 0.21 & 0.61 & 1.91 & 1.91 & 3.22 & 0.97 & 2.76 & 0.11 & 1.19 & 3.90 & 3.90 & 30.93 & 60.18 & 0.11 & 3.09 & $\begin{array}{c}< \\
0.01\end{array}$ & 0.83 & 1.21 & 1.52 & 1.05 & 0.01 & 1.18 & 1.10 & 1.22 & 2.57 \\
\hline $\mathrm{MnO}(\%)$ & 0.01 & 0.27 & 0.01 & 0.01 & 0.01 & 0.12 & 0.01 & 0.08 & 0.46 & 0.27 & 0.02 & 0.02 & 0.06 & 0.09 & 0.04 & 0.06 & 0.01 & 0.17 & 0.03 & 0.27 & 0.15 & 0.04 & 0.20 & 0.18 & 0.31 & 0.02 \\
\hline $\mathrm{MgO}(\%)$ & 0.01 & 20.48 & 1.35 & 2.50 & 2.50 & 3.81 & 0.66 & 5.26 & 21.31 & 7.83 & 2.86 & 2.86 & 11.61 & 1.28 & 1.26 & 5.39 & 0.18 & 8.75 & 2.82 & 5.60 & 3.60 & 0.53 & 4.22 & 3.86 & 5.87 & 3.10 \\
\hline $\mathrm{CaO}(\%)$ & 0.01 & 29.58 & 0.40 & 0.37 & 0.37 & 4.81 & 0.21 & 1.51 & 29.77 & 12.3 & 1.00 & 1.00 & 19.42 & 14.45 & 7.96 & 0.39 & 0.29 & 13.78 & 0.76 & 7.16 & 4.63 & 6.10 & 8.63 & 5.98 & 7.56 & 0.48 \\
\hline $\mathrm{Na}_{2} \mathrm{O}(\%)$ & 0.01 & 0.03 & 1.83 & 0.61 & 0.61 & 0.08 & 0.11 & 1.92 & 0.02 & 0.04 & 0.28 & 0.28 & 0.02 & 0.03 & 0.01 & 1.09 & $<0.01$ & 0.02 & 1.12 & 0.08 & 1.22 & 0.03 & 0.05 & 0.64 & 0.29 & 0.06 \\
\hline $\mathrm{K}_{2} \mathrm{O}(\%)$ & 0.01 & 0.09 & 4.92 & 6.39 & 6.39 & 4.68 & 5.72 & 2.26 & 0.04 & 2.89 & 5.79 & 5.79 & 0.10 & 0.29 & 0.01 & 1.91 & $<0.01$ & 0.32 & 4.60 & 3.19 & 2.81 & 1.34 & 2.75 & 3.82 & 3.00 & 6.73 \\
\hline LOI (\%) & & 46.14 & 1.44 & 3.72 & 3.72 & 8.70 & 1.96 & 4.84 & 46.26 & 18.07 & 3.92 & 3.92 & 29.54 & 14.63 & 30.01 & 6.16 & 21.65 & 19.30 & 4.67 & 13.87 & 5.78 & 11.04 & 12.02 & 5.61 & 11.98 & 6.07 \\
\hline Total (\%) & 0.01 & 98.36 & 99.37 & 99.04 & 99.04 & 100.10 & 100.00 & 99.35 & 99.02 & 98.82 & 100.10 & 100.10 & 98.19 & 100.60 & 40.60 & 96.84 & 28.79 & 82.29 & 97.75 & 92.67 & 98.84 & 65.98 & 95.86 & 95.75 & 97.40 & 95.17 \\
\hline $\mathrm{FeO}(\%)$ & 0.1 & 0.2 & 1.5 & 1.5 & 2.8 & 0.9 & 0.4 & 3.7 & 0.2 & 0.1 & 1.2 & 0.3 & $<0.1$ & $<0.1$ & $<0.1$ & 4.4 & 0.2 & $<0.1$ & 0.9 & 0.6 & 1.3 & 0.2 & $<0.1$ & 1.2 & 0.5 & 0.2 \\
\hline S (\%) & 0.001 & 0.050 & - & - & - & - & - & - & - & - & 0.005 & - & - & - & 0.021 & 0.041 & 1.160 & - & - & - & - & - & - & - & - & - \\
\hline $\mathrm{SO}_{4}(\%)$ & 0.05 & - & - & - & - & - & - & - & - & - & - & - & - & - & - & - & - & 7.80 & - & - & - & - & - & 2.60 & - & - \\
\hline $\mathrm{Sc}(\mathrm{ppm})$ & 1 & $<1$ & 3 & 16 & 14 & 6 & 4 & 24 & $<1$ & 4 & 11 & 3 & 1 & 1 & 3 & 18 & 3 & 1 & 10 & 6 & 10 & 6 & 12 & 10 & 4 & 15 \\
\hline $\mathrm{Be}(\mathrm{ppm})$ & 1 & $<1$ & $<1$ & 4 & 4 & 3 & $<1$ & 1 & $<1$ & 1 & 3 & $<1$ & 1 & 3 & $<1$ & 1 & $<1$ & $<1$ & 3 & 2 & 2 & $<1$ & 1 & 2 & 2 & 4 \\
\hline $\mathrm{V}$ (ppm) & 5 & 15 & 32 & 107 & 96 & 64 & 25 & 177 & 5 & 33 & 76 & 20 & 209 & 313 & 10 & 140 & $<5$ & 24 & 85 & 54 & 64 & 56 & 94 & 59 & 26 & 210 \\
\hline $\mathrm{Cr}(\mathrm{ppm})$ & 20 & $<20$ & 30 & 100 & 80 & 50 & 20 & 90 & $<20$ & 50 & 60 & $<20$ & $<20$ & 30 & $<20$ & 100 & $<20$ & $<20$ & 40 & 80 & 70 & 50 & 50 & 40 & 20 & 90 \\
\hline $\mathrm{Co}(\mathrm{ppm})$ & 1 & 1 & 4 & 3 & 6 & 9 & 5 & 25 & $<1$ & 7 & 12 & 3 & 15 & 35 & 6 & 45 & 20 & 5 & 8 & 12 & 12 & 9 & 7 & 9 & 10 & 37 \\
\hline $\mathrm{Ni}(\mathrm{ppm})$ & 20 & $<20$ & $<20$ & 30 & 50 & 30 & $<20$ & 50 & $<20$ & $<20$ & 40 & $<20$ & 40 & 40 & $<20$ & 70 & $<20$ & $<20$ & $<20$ & $<20$ & $<20$ & $<20$ & $<20$ & $<20$ & $<20$ & 50 \\
\hline
\end{tabular}


Table 1. Cont.

\begin{tabular}{|c|c|c|c|c|c|c|c|c|c|c|c|c|c|c|c|c|c|c|c|c|c|c|c|c|c|c|}
\hline \multirow{2}{*}{\multicolumn{2}{|c|}{ Oxides/Elements }} & \multicolumn{11}{|c|}{ Host Rocks } & \multicolumn{14}{|c|}{ Mineralization } \\
\hline & & \multirow[t]{2}{*}{ 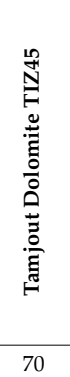 } & \multirow[t]{2}{*}{ 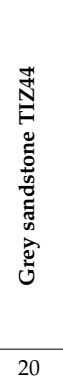 } & \multirow[t]{2}{*}{ 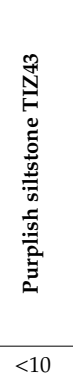 } & \multirow[t]{2}{*}{ 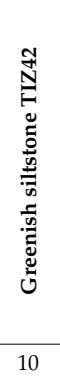 } & \multirow[t]{2}{*}{ 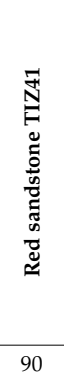 } & \multirow[t]{2}{*}{ 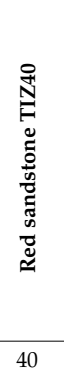 } & \multirow[t]{2}{*}{ 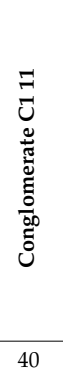 } & \multirow[t]{2}{*}{ 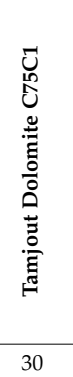 } & \multirow[t]{2}{*}{ 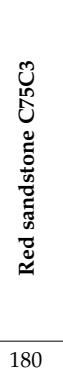 } & \multirow[t]{2}{*}{ 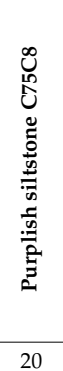 } & \multirow[t]{2}{*}{ 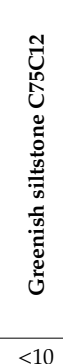 } & \multirow[t]{2}{*}{ 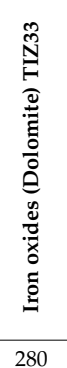 } & \multirow[t]{2}{*}{ 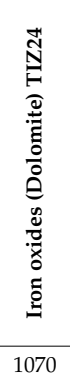 } & \multirow[t]{2}{*}{ 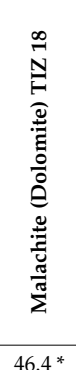 } & \multirow[t]{2}{*}{ 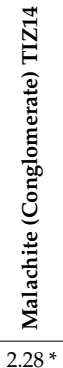 } & \multirow[t]{2}{*}{ 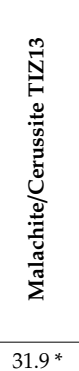 } & \multirow[t]{2}{*}{ 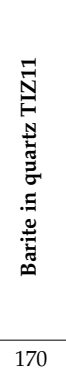 } & 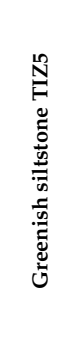 & 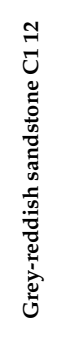 & 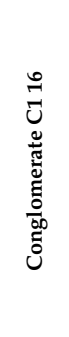 & 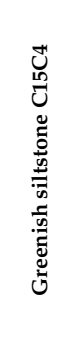 & 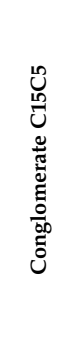 & 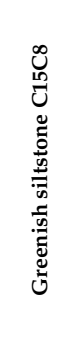 & 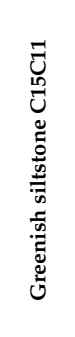 & 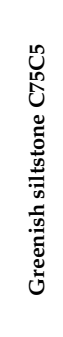 \\
\hline $\mathrm{Cu}(\mathrm{ppm} / \% *)$ & $10 / 0.005$ & & & & & & & & & & & & & & & & & & & $4 *$ & 40 & $30.7 *$ & $2.24 *$ & $3.86^{*}$ & 9290 & $4.23 *$ \\
\hline $\mathrm{Zn}(\mathrm{ppm})$ & 30 & 40 & $<30$ & 30 & 40 & 120 & 110 & 240 & $<30$ & 70 & 70 & $<30$ & 150 & 370 & 530 & 140 & 5990 & 310 & 40 & 90 & 100 & 30 & 30 & 60 & $<30$ & 100 \\
\hline $\mathrm{Ga}(\mathrm{ppm})$ & 1 & 1 & 11 & 29 & 26 & 14 & 10 & 17 & 1 & 11 & 20 & 9 & 2 & 5 & $<1$ & 16 & 11 & 2 & 17 & 14 & 15 & 6 & 11 & 15 & 12 & 28 \\
\hline Ge (ppm) & 1 & $<1$ & 1 & 2 & 2 & 1 & 1 & 1 & $<1$ & $<1$ & 2 & $<1$ & 2 & 14 & $<1$ & 2 & $<1$ & $<1$ & 1 & $<1$ & 1 & $<1$ & $<1$ & 1 & $<1$ & 2 \\
\hline As (ppm) & 5 & 16 & $<5$ & $<5$ & $<5$ & 68 & 17 & 5 & $<5$ & 28 & $<5$ & $<5$ & 419 & 1000 & 32 & $<5$ & 930 & 13 & $<5$ & 9 & 11 & 164 & 166 & 5 & $<5$ & 30 \\
\hline $\mathrm{Rb}$ (ppm) & 2 & 2 & 74 & 253 & 198 & 94 & 91 & 65 & $<2$ & 68 & 156 & 64 & 2 & 8 & $<2$ & 55 & $<2$ & 7 & 114 & 81 & 86 & 30 & 72 & 87 & 80 & 178 \\
\hline $\mathrm{Sr}(\mathrm{ppm})$ & 2 & 85 & 80 & 38 & 53 & 51 & 47 & 56 & 57 & 52 & 38 & 122 & 41 & 45 & 7 & 28 & 307 & 731 & 44 & 94 & 88 & 17 & 62 & 98 & 85 & 24 \\
\hline Y (ppm) & 1 & 2 & 10 & 32 & 26 & 20 & 16 & 13 & 1 & 25 & 17 & 13 & 2 & 7 & 2 & 16 & 1 & 5 & 14 & 31 & 23 & 12 & 40 & 20 & 24 & 18 \\
\hline $\mathrm{Zr}(\mathrm{ppm})$ & 2 & 6 & 70 & 171 & 155 & 171 & 120 & 148 & 4 & 230 & 126 & 104 & 20 & 36 & 6 & 128 & 3 & 26 & 164 & 171 & 419 & 60 & 120 & 141 & 120 & 188 \\
\hline Mo (ppm) & 2 & $<2$ & $<2$ & $<2$ & $<2$ & $<2$ & $<2$ & $<2$ & $<2$ & $<2$ & $<2$ & $<2$ & 73 & 72 & $<2$ & $<2$ & 9 & $<2$ & $<2$ & $<2$ & $<2$ & $<2$ & 3 & $<2$ & $<2$ & 2 \\
\hline In (ppm) & 0.2 & $<0.2$ & $<0.2$ & $<0.2$ & $<0.2$ & $<0.2$ & $<0.2$ & $<0.2$ & $<0.2$ & $<0.2$ & $<0.2$ & $<0.2$ & $<0.2$ & $<0.2$ & $<0.2$ & $<0.2$ & $<0.2$ & $<0.2$ & $<0.2$ & $<0.2$ & $<0.2$ & $<0.2$ & $<0.2$ & $<0.2$ & $<0.2$ & $<0.2$ \\
\hline Sn (ppm) & 1 & $<1$ & 1 & 4 & 3 & 1 & 1 & 1 & 1 & 1 & 3 & 1 & $<1$ & 1 & $<1$ & 1 & 2 & $<1$ & 2 & 1 & 2 & 1 & 1 & 1 & 1 & 3 \\
\hline $\mathrm{Sb}$ (ppm) & 0.5 & 0.6 & $<0.5$ & 2.5 & 1.5 & 3 & 0.5 & $<0.5$ & $<0.5$ & 1.2 & 1.4 & $<0.5$ & 11.3 & 151 & 1.5 & 0.7 & 5.4 & 1.9 & 1 & 0.6 & 0.6 & 15.5 & 1.5 & 0.7 & 0.5 & 2.8 \\
\hline Cs (ppm) & 0.5 & $<0.5$ & 1.5 & 12.6 & 8.9 & 3.5 & 3 & 3 & $<0.5$ & 3.5 & 9.4 & 2.4 & $<0.5$ & $<0.5$ & $<0.5$ & 3 & $<0.5$ & $<0.5$ & 5.5 & 3.6 & 3.7 & 2.1 & 3.3 & 4.4 & 4.5 & 11.9 \\
\hline $\mathrm{Ba}(\mathrm{ppm} / \% *)$ & 2 & 13 & 524 & 614 & 621 & 526 & 633 & 651 & 11 & 334 & 707 & 827 & 67 & 143 & 80 & 1844 & 60 & $10.6^{*}$ & 573 & 8298 & 638 & 188 & 5449 & 841 & 1723 & 737 \\
\hline $\mathrm{Hf}(\mathrm{ppm})$ & 0.2 & $<0.2$ & 1.5 & 4.4 & 3.8 & 3.8 & 2.7 & 3.2 & $<0.2$ & 11.5 & 4 & 2.5 & 0.2 & 1 & $<0.2$ & 3 & $<0.2$ & 0.6 & 3.5 & 3.3 & 8.8 & 1.5 & 2.6 & 3.9 & 2.9 & 5.4 \\
\hline Ta (ppm) & 0.1 & $<0.1$ & 0.3 & 1.1 & 1 & 0.6 & 0.4 & 0.4 & $<0.1$ & 0.6 & 0.7 & 0.4 & $<0.1$ & $<0.1$ & $<0.1$ & 0.4 & $<0.1$ & 0.2 & 0.7 & 0.5 & 0.6 & 0.2 & 0.3 & 0.5 & 0.4 & 1 \\
\hline $\mathrm{W}(\mathrm{ppm})$ & 1 & $<1$ & $<1$ & 2 & 2 & 2 & $<1$ & $<1$ & $<1$ & $<1$ & $<1$ & $<1$ & $<1$ & 2 & $<1$ & $<1$ & $<1$ & 2 & 1 & $<1$ & 2 & 1 & $<1$ & 4 & $<1$ & $<1$ \\
\hline $\mathrm{Tl}$ (ppm) & 0. & $<0.1$ & 0.3 & 0.9 & 0.7 & 0.4 & 0.4 & 0.4 & $<0.1$ & 0.3 & 0.8 & 0.4 & 0.1 & 0.1 & $<0.1$ & 0.3 & $<0.1$ & $<0.1$ & 0.6 & 0.5 & 0.6 & 0.1 & 0.4 & 0.6 & 0.5 & 0.9 \\
\hline $\mathrm{Pb}(\mathrm{ppm} / \% *)$ & $5 / 0.01$ & 316 & 6 & 9 & $<5$ & 10 & 7 & $<5$ & $<5$ & 15 & 6 & 5 & 287 & 551 & 316 & 21 & $29.6 *$ & 213 & $<5$ & 10 & $<5$ & 13 & $<5$ & 107 & $<5$ & 622 \\
\hline $\mathrm{Bi}(\mathrm{ppm})$ & 0.4 & $<0.4$ & $<0.4$ & $<0.4$ & $<0.4$ & $<0.4$ & $<0.4$ & $<0.4$ & $<0.4$ & $<0.4$ & $<0.4$ & $<0.4$ & $<0.4$ & 37.8 & $<0.4$ & $<0.4$ & 0.4 & $<0.4$ & 14 & 6.2 & $<0.4$ & 0.5 & 1.4 & 0.7 & 0.4 & $<0.4$ \\
\hline Th (ppm) & 0.1 & 0.2 & 5.1 & 11.7 & 12.6 & 7.5 & 7.3 & 2.8 & $<0.1$ & 8 & 9.9 & 5.6 & 0.2 & 0.8 & $<0.1$ & 2.1 & $<0.1$ & 0.7 & 8.8 & 6.8 & 8.4 & 2.3 & 2.7 & 4.2 & 6 & 12.5 \\
\hline U (ppm) & 0.1 & 1.3 & 1 & 3.2 & 2.5 & 2.2 & 1.3 & 1.1 & 0.7 & 2 & 2.2 & 1.2 & 4.5 & 13.8 & 0.3 & 0.7 & 0.6 & 2.4 & 1.7 & 2.5 & 2.2 & 2 & 1.7 & 3.2 & 1.8 & 3.8 \\
\hline $\mathrm{La}(\mathrm{ppm})$ & 0.1 & 1.9 & 26 & 58.1 & 56.2 & 31.8 & 24.5 & 8.3 & 1.1 & 26.5 & 32.3 & 28.3 & 3.4 & 6.9 & 1.2 & 11.5 & 1 & 5.7 & 28.8 & 27 & 46.5 & 8.1 & 18.3 & 10.4 & 33.6 & 35.6 \\
\hline Ce (ppm) & 0.1 & 2.5 & 65.7 & 114 & 109 & 61.4 & 52 & 19.3 & 1.5 & 58.6 & 59.5 & 58.7 & 12.4 & 11.1 & 1.5 & 26.1 & 0.9 & 9.9 & 56.9 & 63.9 & 94.1 & 17.8 & 42.7 & 22.7 & 72.4 & 64.9 \\
\hline $\operatorname{Pr}(\mathrm{ppm})$ & 0.05 & 0.39 & 7.25 & 12.7 & 11.6 & 6.91 & 5.81 & 2.37 & 0.2 & 6.87 & 6.25 & 6.21 & 1 & 2.02 & 0.21 & 3.33 & 0.17 & 1.1 & 6.03 & 7.63 & 10.3 & 2.58 & 5.81 & 2.49 & 6.71 & 6.75 \\
\hline $\mathrm{Nd}(\mathrm{ppm})$ & 0.1 & 1.5 & 26.1 & 45.6 & 40.1 & 24.5 & 20.6 & 9.5 & 0.9 & 26.2 & 22.4 & 22.8 & 3.8 & 8.4 & 0.8 & 13.3 & 0.8 & 4.7 & 20.6 & 28.5 & 35.8 & 11.6 & 27 & 10.1 & 23.3 & 23.4 \\
\hline $\mathrm{Sm}(\mathrm{ppm})$ & 0.1 & 0.3 & 4.3 & 8.3 & 6.9 & 4.8 & 3.5 & 2.1 & 0.2 & 5.3 & 4.1 & 3.8 & 0.9 & 1.9 & 0.1 & 3.1 & 0.2 & 1.2 & 3.6 & 4.8 & 6.8 & 2.9 & 8.3 & 2.2 & 4.5 & 4.2 \\
\hline
\end{tabular}


Table 1. Cont.

\begin{tabular}{|c|c|c|c|c|c|c|c|c|c|c|c|c|c|c|c|c|c|c|c|c|c|c|c|c|c|c|}
\hline & & & & & & & st Roc & & & & & & & & & & & & Minera & ization & & & & & & \\
\hline Oxide & & 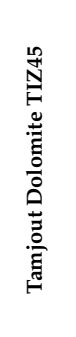 & 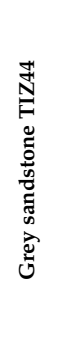 & 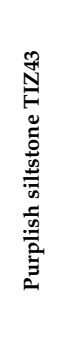 & 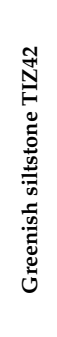 & 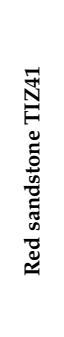 & 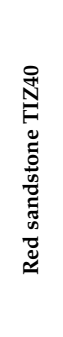 & 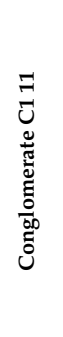 & 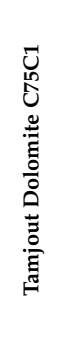 & 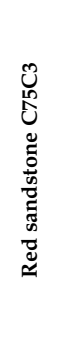 & 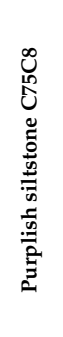 & 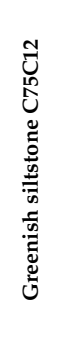 & 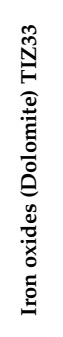 & 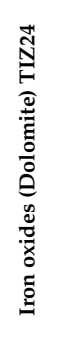 & 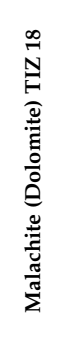 & 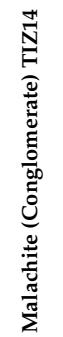 & 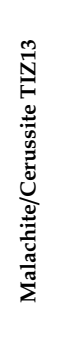 & 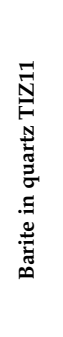 & 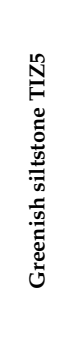 & 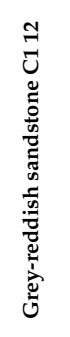 & 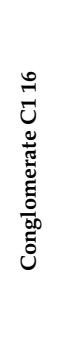 & 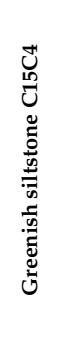 & 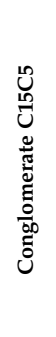 & 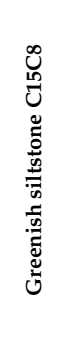 & 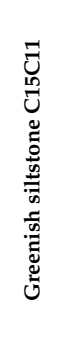 & 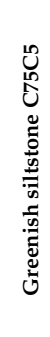 \\
\hline $\mathrm{Eu}(\mathrm{ppm})$ & 0.05 & 0.08 & 0.96 & 1.7 & 1.45 & 1.23 & 0.8 & 0.56 & $<0.05$ & 1.19 & 0.86 & 0.86 & 0.26 & 0.43 & $<0.05$ & 0.93 & 0.06 & 0.99 & 0.68 & 1.02 & 1.59 & 0.77 & 2.3 & 0.61 & 1.22 & 0.85 \\
\hline Gd (ppm) & 0.1 & 0.4 & 2.8 & 6.8 & 5.6 & 4.4 & 3.2 & 2.1 & 0.2 & 4.7 & 3.2 & 2.9 & 0.9 & 1.9 & 0.2 & 3.3 & 0.1 & 1.3 & 2.7 & 4.9 & 5.2 & 2.9 & 10.6 & 2.9 & 4.7 & 3.4 \\
\hline $\mathrm{Tb}$ (ppm) & 0.1 & $<0.1$ & 0.4 & 1.1 & 0.9 & 0.7 & 0.5 & 0.4 & $<0.1$ & 0.7 & 0.5 & 0.4 & 0.1 & 0.3 & $<0.1$ & 0.6 & $<0.1$ & 0.2 & 0.4 & 0.7 & 0.8 & 0.4 & 1.7 & 0.6 & 0.8 & 0.5 \\
\hline Dy (ppm) & 0.1 & 0.3 & 1.8 & 6.1 & 5 & 4 & 3 & 2.4 & 0.1 & 4 & 3.2 & 2.5 & 0.8 & 1.4 & 0.2 & 3.5 & $<0.1$ & 0.7 & 2.6 & 4 & 4.7 & 2.4 & 8.5 & 4 & 5 & 3.3 \\
\hline Ho (ppm) & 0.1 & $<0.1$ & 0.4 & 1.2 & 1 & 0.8 & 0.6 & 0.5 & $<0.1$ & 0.8 & 0.7 & 0.5 & 0.1 & 0.2 & $<0.1$ & 0.7 & $<0.1$ & 0.2 & 0.6 & 0.9 & 0.9 & 0.5 & 1.4 & 0.8 & 1 & 0.7 \\
\hline $\operatorname{Er}(\mathrm{ppm})$ & 0.1 & 0.2 & 1 & 3.5 & 3 & 2.1 & 1.7 & 1.6 & $<0.1$ & 2.2 & 2 & 1.4 & 0.4 & 0.6 & 0.1 & 1.9 & $<0.1$ & 0.4 & 1.6 & 2.4 & 2.7 & 1.3 & 3.3 & 2.3 & 2.6 & 2.2 \\
\hline $\operatorname{Tm}(\mathrm{ppm})$ & 0.05 & $<0.05$ & 0.16 & 0.5 & 0.44 & 0.3 & 0.24 & 0.26 & $<0.05$ & 0.32 & 0.3 & 0.21 & 0.05 & 0.09 & $<0.05$ & 0.28 & $<0.05$ & 0.05 & 0.25 & 0.32 & 0.37 & 0.17 & 0.36 & 0.32 & 0.38 & 0.35 \\
\hline $\mathrm{Yb}(\mathrm{ppm})$ & 0.1 & 0.2 & 1 & 3.4 & 2.9 & 1.9 & 1.6 & 1.9 & $<0.1$ & 2 & 2.1 & 1.5 & 0.3 & 0.4 & 0.1 & 1.8 & $<0.1$ & 0.3 & 1.6 & 2 & 2.5 & 1.1 & 2 & 2.1 & 2.3 & 2.5 \\
\hline $\mathrm{Lu}(\mathrm{ppm})$ & 0.01 & 0.03 & 0.14 & 0.51 & 0.45 & 0.31 & 0.25 & 0.29 & 0.01 & 0.33 & 0.33 & 0.22 & 0.04 & 0.05 & 0.02 & 0.27 & $<0.01$ & 0.05 & 0.24 & 0.3 & 0.37 & 0.17 & 0.28 & 0.3 & 0.33 & 0.41 \\
\hline
\end{tabular}



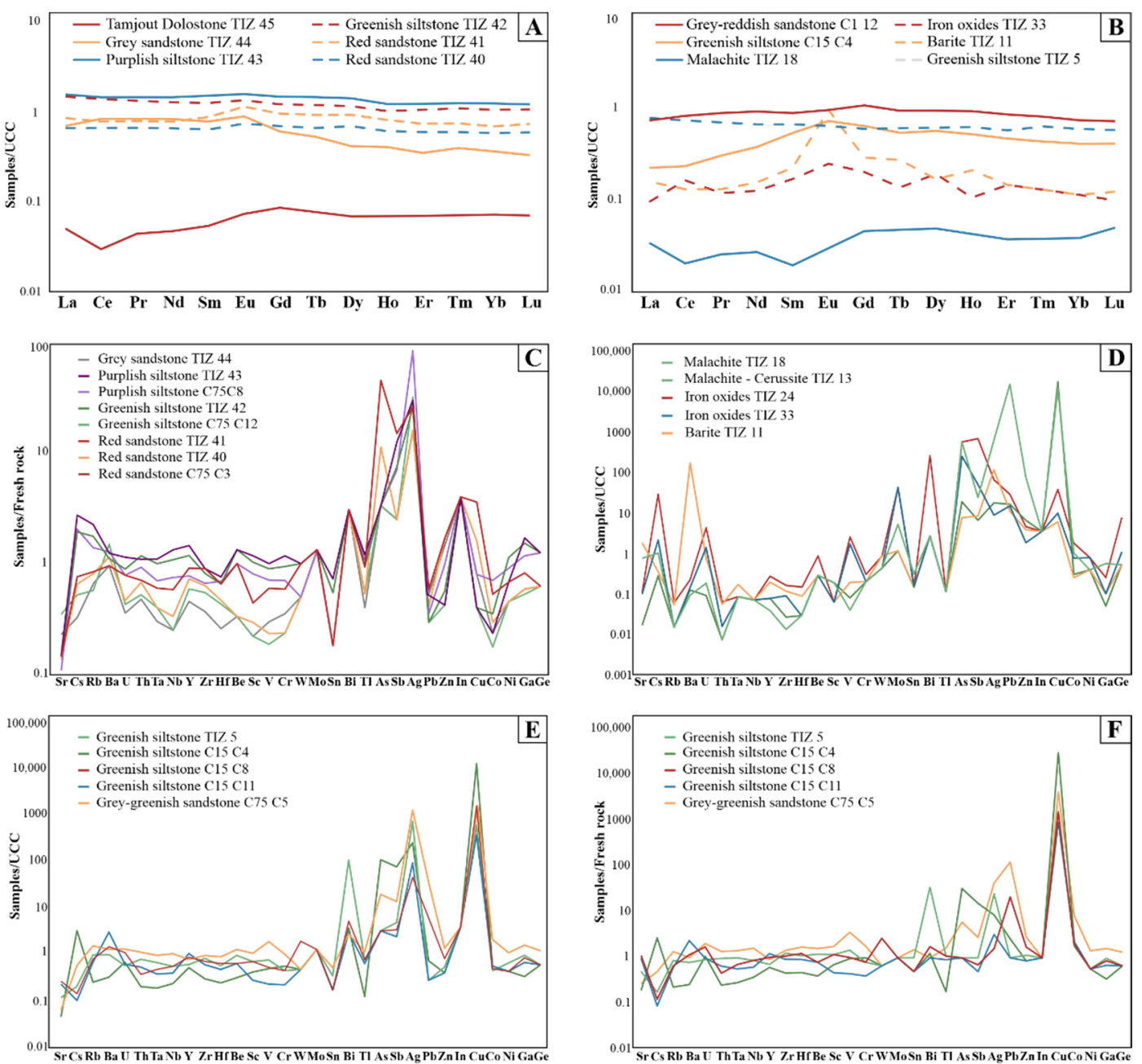

Figure 4. Geochemical data: (A) REE (Rare Earth Elements) patterns for fresh rocks (Tamjout Dolomite and Basal Series), with values normalized to UCC (Upper Continental Crust [44]); (B) REE patterns for weathered samples with values normalized to UCC; $(\mathbf{C})$ trace elements patterns for fresh host rocks (Basal Series), with values normalized to UCC (D) trace elements patterns for main mineral phases in the Tamjout Dolomite, with values normalized to UCC. (E) trace elements patterns for weathered greenish siltstone/sandstone, with values normalized to UCC; $(\mathbf{F})$ trace elements patterns for weathered greenish siltstone/sandstone, with values normalized to fresh greenish siltstone.

The lower part of the Basal Series also contains a Basal Limestone, the so called "Petit Calcaire". This rock (e.g., TIZ 39) is actually a calcareous dolostone composed of dolomite, quartz, calcite and fluorapatite. This unit has a relatively small thickness (a few meters) and it is not observed in the three studied boreholes. Samples of PIII (Precambrian III) conglomerate (Basal conglomerate in Figure 3; e.g., sample C1 19 in Table 1) were also analyzed by XRD. This rock is composed of quartz, calcite, hematite, albite, muscovite, apatite, orthoclase and chlorite and is transitional between the Basal Series and the Tiddiline Formation. 

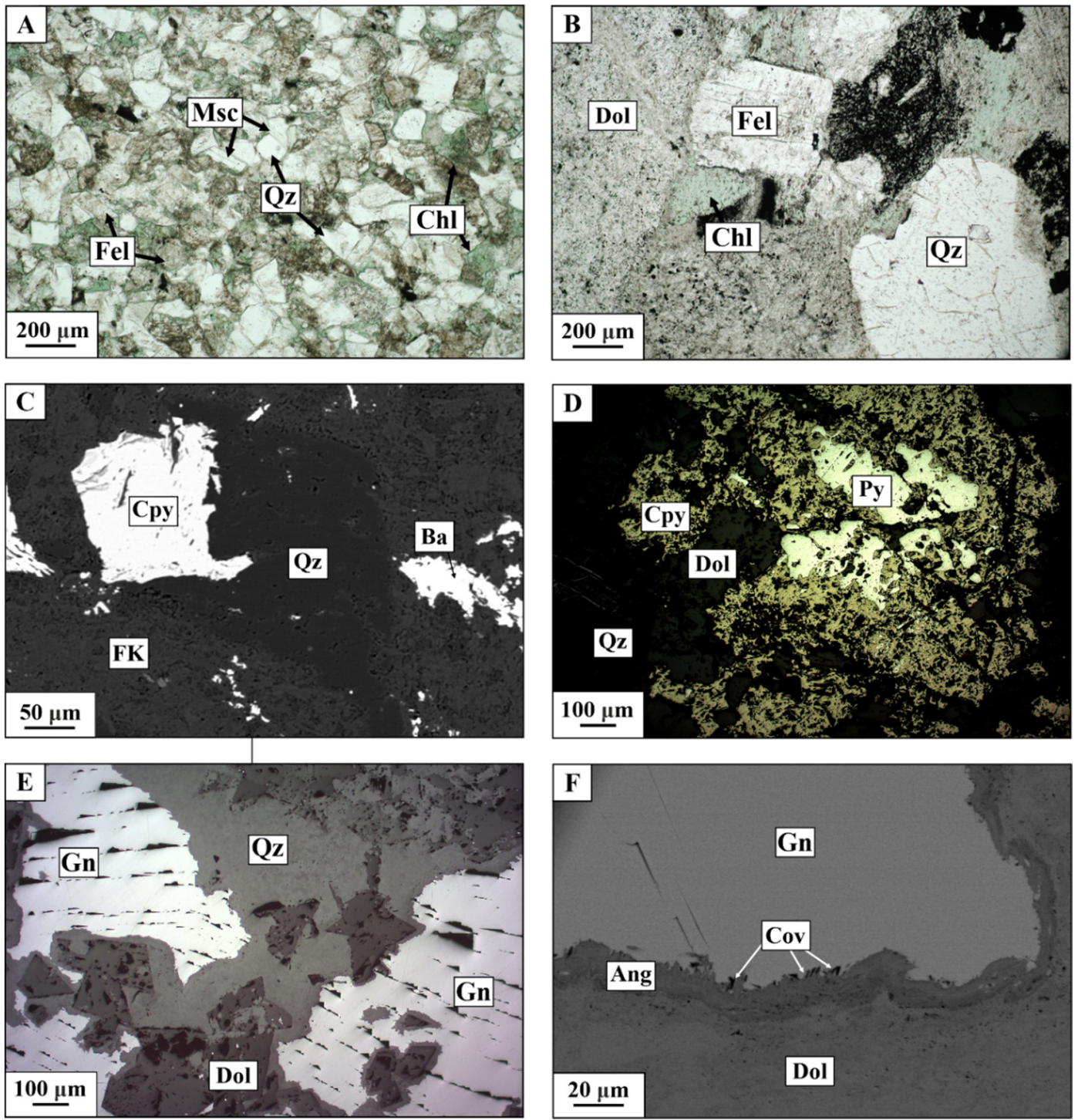

Figure 5. Representative samples and observations of the host rocks: transmitted polarized light microphotographs (A,B), SEM microphotographs in backscattered electrons mode (C,F) and reflected polarized light microphotographs (D,E). (A) Abundance of chlorite (Chl—chlorite; Qz-quartz; Msc-muscovite; Fel-feldspar) in light-grey sandstone (TIZ 44); (B) Red sandstone (TIZ 41); (C) Chalcopyrite (Cpy) and barite (Ba) in coarse grey sandstone (TIZ S19); (D) Pyrite (Py) and chalcopyrite in Basal Conglomerate (TIZ S10); (E) Galena (Gn) in the Tamjout Dolomite (TIZ 45); (F) Galena, covellite (Cov) sticks and anglesite (Ang) in the Tamjout Dolomite (TIZ 45).

\subsection{Primary Mineralization}

The authors in reference [4] described primary mineralization to be composed of sulfides such as chalcopyrite, pyrite, bornite I, chalcocite I and galena. These primary sulfides were observed in samples TIZ S5 (sandstone), S6 (grey sandstone), S10 (conglomerate) and S19 (coarse sandstone). Bornite (e.g., TIZ S6) and chalcopyrite (e.g., TIZ S5 and S19, Figure 5C) are mostly observed separately, with exceptions made for some samples (e.g., TIZ S10, Figure 5D). These primary sulfides are disseminated in the host rock (sandstone and conglomerate of the Basal Series, and Lower Limestone) and are rare or absent in subsurface samples due to weathering. SEM analyses reveal low values $(<0.5 \mathrm{wt} . \%)$ of $\mathrm{As}, \mathrm{Pb}$ (except for galena), $\mathrm{Zn}$ and other chalcophile elements in $\mathrm{Cu}$-sulfides. The Tamjout Dolomite (TIZ 45) shows a very slight mineralization characterized by the presence of galena (Figure 5E,F). These primary sulfides, such as pyrite and chalcopyrite, are relatively homogeneous and 
do not contain significant amounts of other metals. Only small variations in terms of $\mathrm{Ag}$ (1.6-0.6 ppm), $\mathrm{Pb}(316-5 \mathrm{ppm})$ and As (16-5 ppm) can be mentioned between TIZ 45 (poorly mineralized) and C75 C1 (fresh) dolostone, which are probably related to a low content of Ag, or even As, in the galena.

\subsection{Secondary (Weathered) Mineralization}

\subsubsection{Tamjout Dolomite}

Malachite is the predominant secondary mineral in the Tamjout Dolomite. Various weathered samples were analyzed; malachite ( $\mathrm{Cu} 31.6 \mathrm{wt} . \%)$ and cerussite ( $\mathrm{Pb} 29.6 \mathrm{wt} . \%)$ could be almost completely isolated from one sample (TIZ 13), except for a small amount of quartz $\left(\mathrm{SiO}_{2} 6.4 \mathrm{wt} . \%\right.$, Table 1). These samples also show relatively high contents of Zn (5990 ppm), As (930 ppm), Sr (307 ppm) and $\mathrm{Ag}(34.5 \mathrm{ppm})$. Chalcocite II is a minor component (1.2 wt.\% S in this sample; Table 1). The REE pattern is similar to the "fresh" Tamjout Dolomite. The Tamjout Dolomite sample (TIZ 45) shows, in low proportions, some secondary mineralization such as anglesite, cerussite and covellite surrounding galena (Figure $5 \mathrm{~F}$ ). Pb-arsenates are also locally observed.

Malachite with some calcite could be isolated (TIZ 18, Figure 6A). In this sample, secondary sulfides such as covellite or chalcocite II are rare, as confirmed by the very low $\mathrm{S}$ content $(\sim 0.02 \mathrm{wt} . \%$; Table 1). An enrichment in $\mathrm{Zn}$ (530 ppm), $\mathrm{Pb}$ (316 ppm) and As (32 ppm) is observed, while Ag content (1 ppm, probably linked to galena) is low compared to the previous sample (TIZ 13). The REE profile is similar to all other Tamjout Dolomite samples (Figure 4B). Small proportions of kaolinite have been identified in some borehole samples (TIZ C15 C3, TIZ C1 A and B). In the upper part of the Tamjout Dolomite, some cubic remnants of pyrite (Figure 6B), locally pseudomorphosed in iron-oxyhydroxides, are observed in quartz veins.

Hematite and goethite associated to calcite, dolomite and quartz are identified within the weathered Tamjout Dolomite. The composition of these samples is mainly controlled by $\mathrm{Fe}_{2} \mathrm{O}_{3}, \mathrm{MgO}, \mathrm{CaO}$ and $\mathrm{SiO}_{2}$ (e.g., TIZ 24 and TIZ 33; Figure 6C; Table 1). V is enriched in some weathered samples (TIZ 24 and TIZ 33, 313 and 209 ppm, respectively) compared to fresh rocks. Enrichments in $\mathrm{Cu}$ (1070 and 280 ppm), As (1000 and 419 ppm), Pb (551 and 287 ppm), Zn (370 and 150 ppm), Sb, Mo, Bi and U are highlighted (Table 1, Figure 4D). No significant enrichment in Ag is detected in iron oxides. The REE pattern is flat and depleted compared to UCC values (Figure 4B). Iron oxyhydroxides are not frequent in the Tamjout Dolomite compared to widespread malachite. In most samples of the Tizert deposit, $\mathrm{Pb}$ content is correlated to As and Zn enrichments (e.g., TIZ 13, TIZ 18, TIZ 24, TIZ 33 and TIZ 45; Table 1).

SEM and transmitted light optical microscopy analyses of the upper part of the Tamjout Dolomite (Figure 6D, S0) close to the Lower Limestone level (Figure 2) reveal the presence of malachite, chicken-wire chalcocite II and covellite, and residual primary chalcopyrite (Figure 6E). Chalcocite II and covellite are observed within malachite (mostly on the edges). The EDS analysis shows small proportions of As in malachite ( 1 wt.\%). Residual chalcopyrite with chalcocite II on the edge is also observed.

\subsubsection{Basal Series}

In the Tizert deposit, most of the mineralization is located in the greenish siltstone of the Basal Series [4]. Most samples located in the central and lower parts of the Basal Series are generally less affected by weathering, and thus, have lower $\mathrm{Cu}$ content. Surface and borehole samples were, therefore, analyzed in this part of the Basal Series. For most of them, secondary Cu-mineralization is disseminated. For example, sample TIZ 5 contains malachite and chemical analysis reveals $1.5 \mathrm{wt} . \% \mathrm{Cu}$ (whole rock) and $36.3 \mathrm{ppm}$ of $\mathrm{Ag}$ (up to $700 \times \mathrm{UCC}$, Table 1, Figure 4D). Ba content is similar to the UCC, while Pb and As are under the detection limit ( $<5$ ppm). Other samples (e.g., TIZ C15 C8 and C11, Figure 4E,F) display higher $\mathrm{Cu}$ content (up to $4 \mathrm{wt} . \%$ ) with variable $\mathrm{Ag}$, $\mathrm{As}, \mathrm{Pb}$ and Ba content (whole rock, Table 1). No major Ag-Pb-As-bearing secondary mineral phases have been identified in these rocks. 

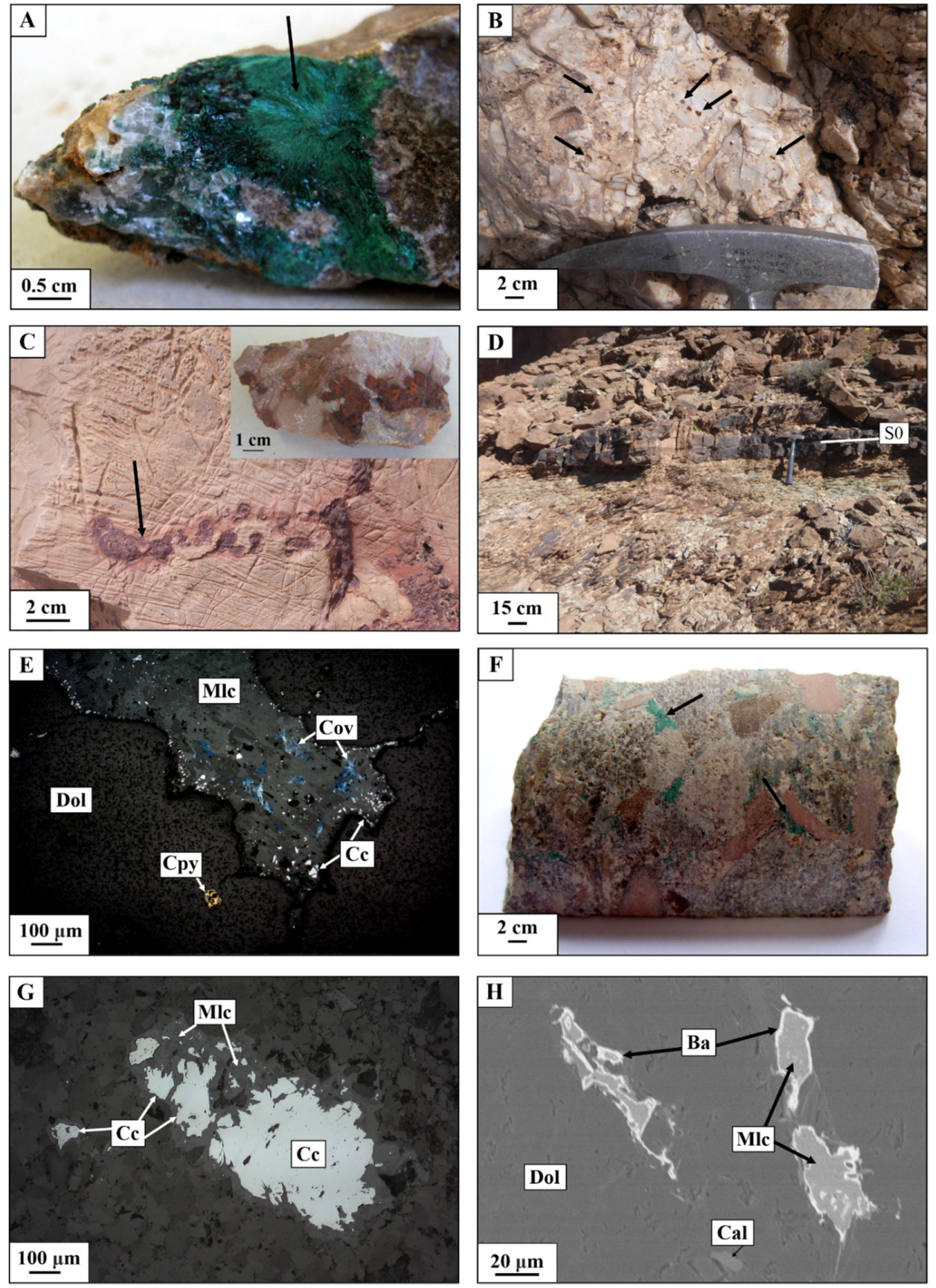

Figure 6. Images of typical weathered materials of the Tizert deposit: field and samples pictures (A-D,F) reflected polarized light microphotographs $(\mathrm{E}, \mathrm{G})$ and SEM microphotographs in the backscattered electrons mode (H). (A) Malachite (Mlc) and calcite (Cal) in the Tamjout Dolomite (TIZ 18); (B) Pyrite dissolution ghosts in quartz veins in the Tamjout Dolomite; (C) Hematite in the Tamjout Dolomite (TIZ 33); (D) Stratification plan (S0) in the Tamjout Dolomite; (E) Malachite, chicken-wire chalcocite (Cc), covellite (Cov) and chalcopyrite (Cpy) in the Tamjout Dolomite (Dol-dolomite, C15 C1); (F) Malachite in matrix of Basal Conglomerate (C15 C5); (G) Malachite and chalcocite in greyish red sandstone in Basal Series (C1 12); (H) Malachite surrounded by barite (Ba) in greyish red sandstone from Basal Series (C1 12).

Only few iron oxides are observed in chalcocite II and could explain slight variations of $\mathrm{Pb}$ and $\mathrm{As}$. In most cases, chalcocite II is surrounded by malachite; rare fluorapatite is concentrated near malachite. Variations for most minor and trace elements in greenish siltstones are generally not consistent with 
fluctuations of the $\mathrm{Cu}$ grade (except for As in malachite). However, high $\mathrm{Ag}$ values are usually related to high $\mathrm{Pb}$, As and Zn contents (e.g., TIZ C75 C5). The REE profile is similar to "fresh" greenish siltstones (Figure 4B).

Conglomerates and microconglomerates also host $\mathrm{Cu}$ mineralization mainly represented by malachite in the matrix (Figure 6F). The matrix of these samples shows up to $2.3 \mathrm{wt} . \%$ of $\mathrm{Cu}$ and low Ba enrichment resulting from the common presence of barite (e.g., TIZ C15 C5 and TIZ 14; Figure 6F; 5549 and 1844 ppm, respectively; Table 1).

Many mineral phases, including secondary mineralization, are identified in greyish red sandstones (e.g., TIZ C1 12, Figure 6G,H): quartz, dolomite, malachite, chalcocite II, hematite, feldspars, apatite (1.3 wt.\% $\left.\mathrm{P}_{2} \mathrm{O}_{5}\right)$, muscovite and barite. It is a sandstone rich in carbonates (low $\mathrm{SiO}_{2}$ content, $47.6 \mathrm{wt} . \%$, Table 1), in which malachite can be surrounded by a rim of barite (Figure $6 \mathrm{H}$ ). The edge of chalcocite II is replaced by malachite (Figure 6G) and hematite. Bornite is also identified inside and outside the malachite. $\mathrm{Cu}$ content for this sample is about $4 \mathrm{wt} . \%$, in addition to $\mathrm{Ba}$, Ag and $\mathrm{Zn}$ enrichments. However, $\mathrm{Pb}$ and As concentrations are very low (10 and 9 ppm, respectively). The REE profile is, again, similar to all samples from the Basal Series, with a slight depletion in LREE (Light Rare Earth Elements, Figure 4D). Basic mineral phases are identified (TIZ C15 C9), such as quartz, muscovite, dolomite, apatite, kaolinite, chlorite, hematite, goethite and calcite. Rare phosphates are rich in $\mathrm{Y}(\mathrm{O}$ 63.8 wt.\%, P 18.1 wt.\% and Y 13.5 wt.\%) and HREE (Dy 1.9 wt.\%, Gd 0.8 wt.\%, Yb 0.9 wt.\% and Er $1.1 \mathrm{wt.} \%)$. The authors in reference [4] described Ag-bearing minerals, such as stromeyerite, eugenite, arquerite $(\mathrm{Ag}, \mathrm{Hg})$, chlorargyrite or native $\mathrm{Ag}$. These minerals were not identified in this study, except in the greenish-grey sandstone (TIZ C75 C7), where associated S, Ag and Cu could be attributed to stromeyerite embodied in malachite. Some barite (surrounding malachite) and anatase $\left(\mathrm{TiO}_{2}\right)$ are also highlighted.

Some clays are observed in the weathered Basal Series, but rarely in the Tamjout Dolomite. These are mainly kaolinite (e.g., TIZ C1 A), illite, smectites (e.g., TIZ C15 C11), chlorite (swelling or not, e.g., TIZ 14, TIZ C1 16) and interstratified clays (mostly illite-smectite, e.g., TIZ C1 12-16). Table 2 summarizes the mineral phases observed in the studied samples.

\section{Discussion}

\subsection{Weathering Profile}

The presence of an oxidizing environment and fluids leaching the primary ore are the main factors leading to the formation of weathered ores $[2,3,45,46]$. Other factors, such as uplift of the area and fracturing, enable the exhumation and subsequent weathering of host rocks and mineralization [2]. The structure, the porosity and the permeability of the host rocks and the presence of faults/fractures facilitate the percolation of meteoric waters, and therefore, control the flow of supergene fluids $[3,47]$. In addition, the nature of the host rocks (e.g., limestone or dolostone) influences neutralization processes of acidic fluids $[2,48,49]$. The weathering profile can be separated into different zones. The upper part of the weathering profile, generally referred to as laterite, gossan or the leaching zone, is rarely conserved in the Tizert deposit and is limited to few subsurface samples in which iron oxyhydroxides are abundant (e.g., TIZ 24, 33 and 36). Below this leaching zone, the oxidation zone (saprolite), also defined as the "green oxide zone", is separated from the cementation zone by the water table level [1]. The limit between the cementation and the oxidation zone reflects the transition from a reducing to an oxidizing environment, allowing the formation of secondary carbonates such as malachite that are stable under oxidizing conditions and for a neutral to basic $\mathrm{pH}$ (Figure 7A) [45,50,51]. Other minerals, such as cerussite (Figure 7B; PbCO3, e.g., TIZ13), sulfates (barite, e.g., TIZ11) or phosphates, may also be formed [51], as is the case at Tizert (Figures $5 \mathrm{C}$ and $6 \mathrm{H}$ ). The cementation zone (saprolite) is characterized by the replacement of primary sulfides by other secondary sulfides [45]. At Tizert, chalcopyrite and bornite I are replaced by chalcocite II, bornite II and covellite, which are stable under more reducing and lower $\mathrm{pH}$ conditions (Figure 7A). These secondary minerals are formed 
by the recombination of metal cations and sulfate ions transported by acidic fluids [52]. The presence of these sulfides indicates that neutralization of the fluids was not sufficient to reach a neutral $\mathrm{pH}$ and that these solutions were able to reach the level of the groundwater table as a redox barrier (transition between oxidizing and reducing conditions) [45,51]. The main Cu minerals are found in the "green oxide zone" (mainly malachite) and in the cementation zone (covellite and chalcocite II). In this part of the weathering profile, also called saprolite, the weathering is less developed; therefore, the original stratification $\left(\mathrm{S}_{0}\right)$ and schistosity are preserved (Figure 6D).
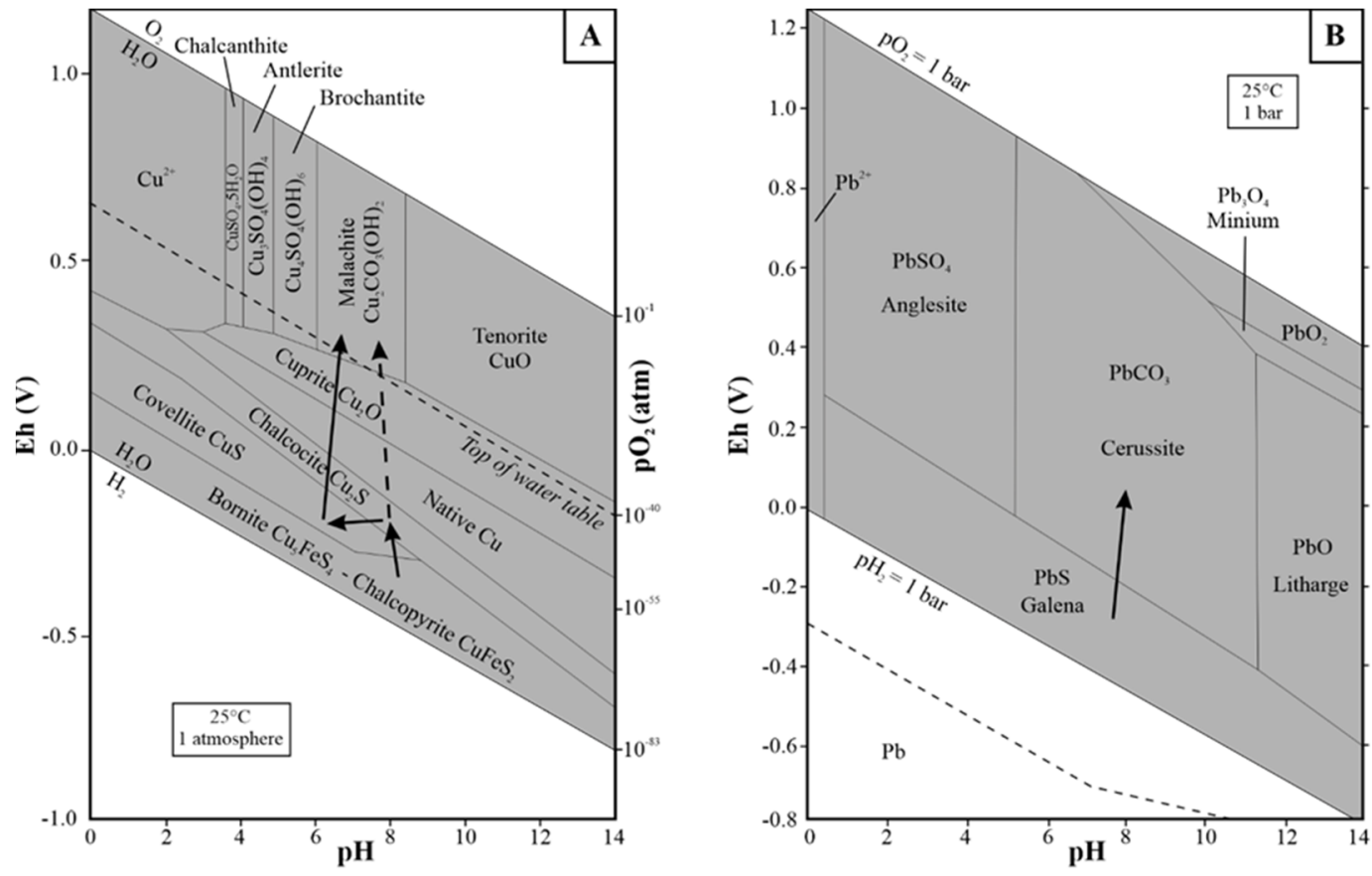

Figure 7. (A) Eh-pH diagram of the system Cu-C-S-Si-O-OH showing stability fields of Cu-minerals at $25^{\circ} \mathrm{C}$ and $1 \mathrm{~atm}$ (modified from reference [53]); (B) Eh-pH diagram of the system Pb-S-C-O-OH showing the stability fields of $\mathrm{Pb}$-minerals at $25^{\circ} \mathrm{C}$ and 1 bar (modified from reference [54]).

\subsection{Paragenesis}

The mineralizing sequence (Figure 8) observed in the Tizert copper deposit is as follows: chalcopyrite-pyrite-bornite I-chalcocite I-galena $\rightarrow$ bornite II $\rightarrow$ chalcocite II/covellite $\rightarrow$ malachite/cerussite $\rightarrow$ hematite, goethite. Primary sulfides (Figure 5CF; chalcopyrite, pyrite, galena, chalcocite I and bornite I) are initially replaced by secondary sulfides (Figure 6E,G; chalcocite II, covellite and bornite II) in the cementation zone. Isolated bornite (e.g., TIZ S6) is primary, while it also could be secondary in some other samples (e.g., TIZ C1 12). Covellite has a smaller stability range than bornite, and chalcopyrite is formed after chalcocite II or directly from chalcopyrite (in some cases, chalcocite II is not observed), since this Cu-depleted sulfide is the last secondary sulfide to precipitate [55]. The presence of covellite reflects slight variations in the groundwater table, leading to more reducing conditions [3,46]. Malachite forms when $\mathrm{pH}$ rises (neutral to basic); this mineral is more abundant in the Lower Limestone because fluid neutralization has been more efficient in carbonate rocks. 


\begin{tabular}{|c|c|c|c|}
\hline \multirow{2}{*}{ MINERALS } & \multirow{2}{*}{ HYPOGENE* } & \multicolumn{2}{|c|}{ SUPERGENE MINERALIZATION } \\
\hline & & CEMENTATION ZONE & SAPROLITE AND LATERITE \\
\hline Chalcopyrite* & & & \\
\hline Bornite I* & & & \\
\hline Bornite II & & & \\
\hline Pyrite* & & & \\
\hline Galena* & & & \\
\hline Chalcocite I* & & & \\
\hline Chalcocite II & & & \\
\hline Covellite & & & \\
\hline Stromeyerite I* & & & \\
\hline Stromeyerite II & & & \\
\hline Malachite & & & \\
\hline Cerussite & & & \\
\hline Barite I & & & \\
\hline Barite II & & & \\
\hline Hematite/Goethite & & & \\
\hline Clays & & & \\
\hline
\end{tabular}

Figure 8. Paragenetic sequence of the Tizert deposit (hypogene and supergene mineralization; * observed by the authors in reference [4]).

Barite is identified as primary along with primary sulfides (e.g., TIZ S19, Figure 5C) and as a secondary mineral forming a rim around malachite (e.g., TIZ C1 12, Figure $6 \mathrm{H}$ ). Barite may precipitate in a large field of $\mathrm{pH}$ and Eh under supergene conditions [56]. At Tizert, the Ba required for the formation of supergene barite probably originates from sandstones and siltstones of the Basal Series that contain a significant quantity of this element (Table 1, 500-800 ppm). As previously proposed by the authors in reference [4], at least two origins can be observed (primary and secondary) for barite at Tizert.

\subsection{Weathering Processes}

Weathering processes are responsible for the in situ supergene enrichment of copper and other elements such as zinc, silver or gold in many near-surface deposits [1,51]. For the Tizert copper deposit, it is mainly an enrichment in $\mathrm{Cu}$ and, to a lower extent, in $\mathrm{Ag}$ [4]. This statement is attributed to three main processes: (1) oxidation of primary sulfides, (2) per descensum transport of acidic solutions and (3) neutralization of acidic fluids, leading to the formation of secondary minerals.

The first step leading to enrichment is the oxidation of the primary sulfides like chalcopyrite, pyrite or bornite I (Figure 9, Equations (1) to (3) [45,51]):

$$
\mathrm{CuFeS}_{2(\mathrm{~s})}+17 / 4 \mathrm{O}_{2(\mathrm{aq})}+10 / 4 \mathrm{H}_{2} \mathrm{O} \rightarrow \mathrm{Fe}(\mathrm{OH})_{3}+\mathrm{Cu}^{2+}{ }_{(\mathrm{aq})}+2 \mathrm{H}^{+}{ }_{(\mathrm{aq})}+2 \mathrm{SO}_{4}{ }^{2-}{ }_{(\mathrm{aq})}
$$




$$
\begin{gathered}
\mathrm{FeS}_{2(\mathrm{~s})}+15 / 2 \mathrm{O}_{2(\mathrm{aq})}+7 / 2 \mathrm{H}_{2} \mathrm{O} \rightarrow \mathrm{Fe}(\mathrm{OH})_{3}+4 \mathrm{H}^{+}{ }_{(\mathrm{aq})}+2 \mathrm{SO}_{4}{ }^{2-}{ }_{(\mathrm{aq})} \\
\mathrm{Cu}_{5} \mathrm{FeS}_{4(\mathrm{~s})}+17 / 4 \mathrm{O}_{2(\mathrm{aq})}+10 / 4 \mathrm{H}_{2} \mathrm{O} \rightarrow \mathrm{Fe}(\mathrm{OH})_{3}+5 \mathrm{Cu}^{2+}{ }_{(\mathrm{aq})}+4 \mathrm{H}^{+}{ }_{(\mathrm{aq})}+4 \mathrm{SO}_{4}{ }^{2-}{ }_{(\mathrm{aq})}
\end{gathered}
$$
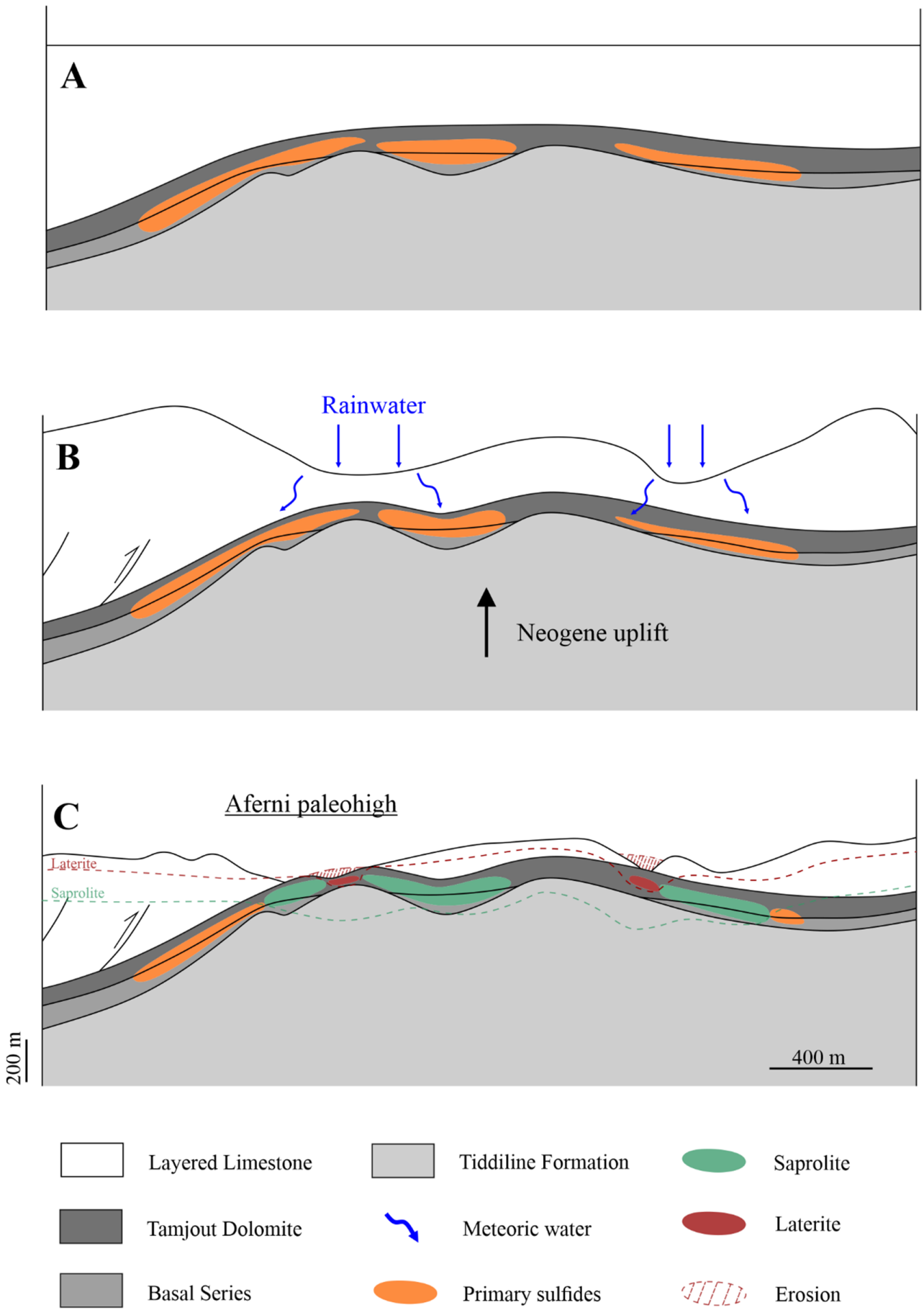

Figure 9. Schematic representation of the weathering model for the supergene Tizert deposit: (A) initial situation (pre- 30 Ma), with fresh primary (hypogene) ore well-developed along basin margins adjacent to the basement paleohighs—see reference [4] for details; (B) initiation of weathering during Neogene uplift(s); (C) current situation, with weathering profile (laterite s.s.-saprolite-primary sulfides) (Modified from reference [4]). 
Table 2. Mineral phases observed in the different samples of the Tizert deposit (H—Host rocks; W—Weathered rocks; XXX—major; $X X-$ frequent; $X-$ minor)

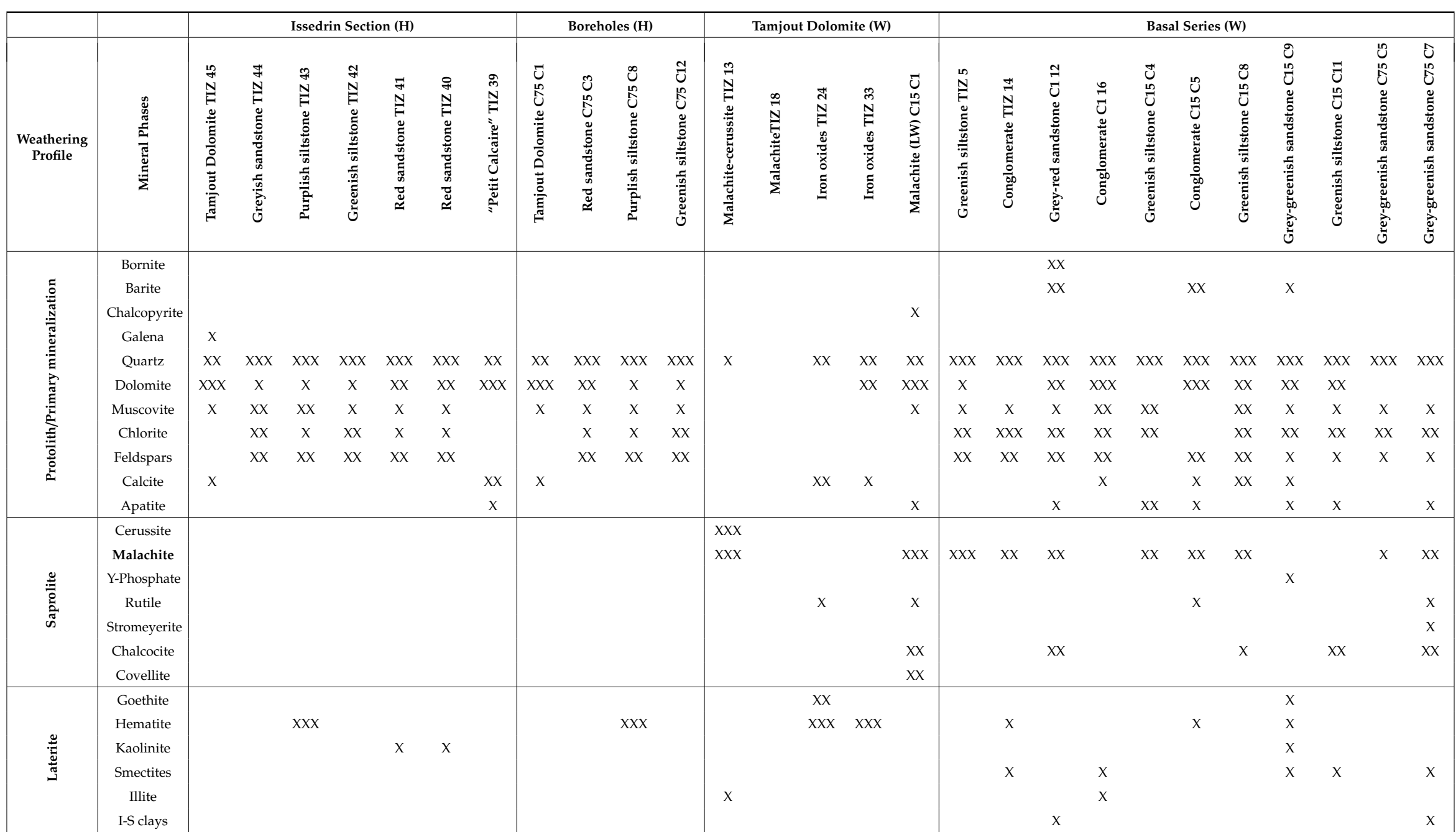


The oxidation of hypogene minerals leads to the production of $\mathrm{H}^{+}$ions (decrease in $\mathrm{pH}$ ) in the weathering zone as well as the release of $\mathrm{SO}_{4}{ }^{2-}$ ions, which will reinforce the weathering process, and other ligands $\left(\mathrm{CO}_{3}{ }^{2-}, \mathrm{PO}_{4}{ }^{3-}, \ldots\right)$ and metallic cations $\left(\mathrm{Cu}^{2+}, \mathrm{Fe}^{2+}, \ldots\right)[1,46,57]$. The hypogene orebody was leached from $\mathrm{Cu}^{2+}$ cations that led to a slight enrichment in Fe and to the formation of hematite or goethite in the upper part of the weathering profile [51]. Such concentration is mainly due to the very low mobility of iron, which is, therefore, not transported by supergene fluids [45]. Other elements (like $\mathrm{Pb}$ and $\mathrm{Zn}$ ) with low mobility can also be concentrated in these lateritic Fe oxides, as is the case in Tizert (e.g., TIZ 24 and TIZ 33, Figure 6C).

Transport is an important step in the enrichment process; once oxidized and leached, the metals-here, mainly $\mathrm{Cu}$ and $\mathrm{Ag}$ - are transported per descensum by acidic fluids over a distance of several tens or even hundreds of meters due to the high mobility of copper $[1,45,46,58]$. Then, neutralization of acidic fluids is necessary to allow the precipitation of secondary minerals that require neutral $\mathrm{pH}$ conditions such as malachite or cerussite (Equations (4) and $(5)[59,60]$, Figure $6 \mathrm{~A}, \mathrm{E}-\mathrm{H})$. This neutralization (Equations (6) to (8)) involves carbonates [59] such as those from the Tamjout Dolomite and/or chlorites [60], the latter being widely available in sandstones and siltstones of the Basal Series (Figure 5A,B). The presence of smectite and/or kaolinite in some samples (e.g., TIZ 3, TIZ C1 C and TIZ C1 10) confirms that part of the fluid has been locally neutralized by chlorites [60]. However, significant amounts of chlorite are still present in the weathering profile. This, in addition to the relative abundance of malachite, suggests that acidic fluids were mainly neutralized by carbonates in the Lower Limestone (especially Tamjout Dolomite) and that the chlorite-rich upper part of the Basal Series only slightly contributed to the neutralization of acidic fluids.

$$
\begin{aligned}
& 2 \mathrm{Cu}^{2+}{ }_{(\mathrm{aq})}+2 \mathrm{H}_{2} \mathrm{O}+\mathrm{HCO}_{3}{ }^{-}{ }_{(\mathrm{aq})} \rightarrow \mathrm{Cu}_{2} \mathrm{CO}_{3}(\mathrm{OH})_{2(\mathrm{~s})}+3 \mathrm{H}^{+}{ }_{(\mathrm{aq})} \\
& \mathrm{PbS}_{(\mathrm{s})}+2 \mathrm{O}_{2(\mathrm{aq})}+\mathrm{HCO}_{3}{ }^{-}{ }_{(\mathrm{aq})} \rightarrow \mathrm{PbCO}_{3(\mathrm{~s})}+\mathrm{SO}_{4}{ }^{2-}{ }_{(\mathrm{aq})}+\mathrm{H}^{+}{ }_{(\mathrm{aq})} \\
& \mathrm{CaCO}_{3(\mathrm{~s})}+\mathrm{H}+{ }_{(\mathrm{aq})} \rightarrow+\mathrm{Ca}^{2+}{ }_{(\mathrm{aq})}+\mathrm{HCO}_{3}{ }_{(\mathrm{aq})} \\
& \mathrm{HCO}_{3}{ }^{-}{ }_{(\mathrm{aq})}+\mathrm{H}^{+}{ }_{(\mathrm{aq})} \rightarrow \mathrm{H}_{2} \mathrm{O}+\mathrm{CO}_{2(\mathrm{~g})} \\
& \text { chlorite }+2 \mathrm{n} \mathrm{H}^{+} \rightarrow \text { smectite or kaolinite }+\mathrm{n}\left(\mathrm{Mg}^{2+}, \mathrm{Fe}^{2+}\right)+\mathrm{m} \mathrm{H}_{2} \mathrm{O}
\end{aligned}
$$

Azurite and malachite have very similar chemical compositions. Malachite is more often observed than azurite because it is more stable under atmospheric conditions [45]. The absence or the very low occurrence of azurite at Tizert can be explained by several factors. The formation of this mineral requires slightly acidic conditions with high carbonate ion activity, while malachite precipitates under neutral to slightly basic $\mathrm{pH}$ [61]. In addition, azurite often recrystallizes into malachite when the activity of carbonate ions slightly varies. However, no pseudomorphosed malachite was observed at Tizert, suggesting that malachite was the only secondary carbonate to precipitate during weathering processes. The transition from malachite to azurite is very sensitive to slight chemical variations in the environment [61]. In the $\mathrm{Cu}-\mathrm{O}-\mathrm{H}-\mathrm{CO}_{2}$ system (Figure 10), the balance between azurite and malachite is reached for a $\mathrm{CO}_{2}$ partial pressure of $10^{-3.45} \mathrm{~atm}$, close to atmospheric pressure; both minerals may coexist at such pressure and at $\mathrm{pH}$ levels ranging from 6.95 to 13.05 [61]. At Tizert, such chemical conditions were not reached and jeopardized the neoformation of azurite. Hence, azurite remains absent (or rarely observed, according to reference [4]). 


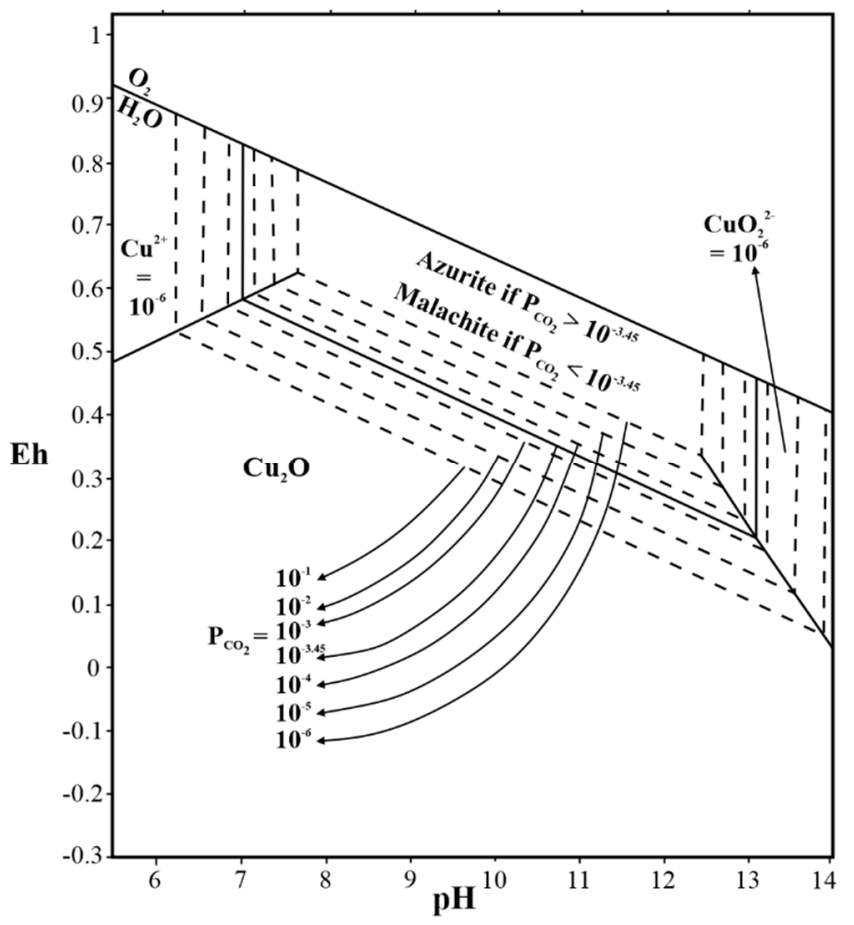

Figure 10. Eh-pH diagram of the system $\mathrm{Cu}-\mathrm{O}-\mathrm{H}-\mathrm{CO}_{2}$ at $25^{\circ} \mathrm{C}$ and $1 \mathrm{~atm}$. total pressure. Total dissolved copper $10^{-6} \mathrm{~mol}$. The diagram shows the stability fields of malachite and azurite as a function of $\mathrm{P}_{\mathrm{CO} 2}$. Equilibrium is reached at $\mathrm{P}_{\mathrm{CO} 2}=10^{-3.45} \mathrm{~atm}$. with corresponding $\mathrm{pH}=6.95, \mathrm{pH}=13.05$ and Eh $=0.67-0.0592 \mathrm{pH}$ (straight lines) (modified from reference [61]).

The absence/scarcity of azurite is quite common in supergene copper deposits of Morocco such as the Jbel N'Zourk deposit (Central Anti-Atlas), for which a similar mineralogical assemblage (except for some Cu-oxides) in the same host rocks (Basal Series-Tamjout Dolomite) is observed [62,63], and the Jbel La'Sal deposit [64,65] in Bou Azzer-El Graara inliers (Table 3), or elsewhere in the world (e.g., Luiswishi deposit, DRC) [66]. On the contrary, other deposits located in the Anti-Atlas, such as Agoujgal, Tazalaght or Ouansimi (Table 3) $[5,6,8,9,67]$, show higher proportions of azurite, even in quite similar host rocks than at Tizert. This is also the case in the Yangla deposit (China) [68] and the Tsumeb deposit (Namibia) [69]. Even if these mineralizations are located in the same host rocks, the local geodynamic context (especially fracturing and weathering depth) of each deposit may have been slightly different and may have locally displayed higher local $\mathrm{pCO}_{2}$, allowing azurite formation. The presence of azurite in supergene $\mathrm{Cu}$-deposits may be related to the formation of carbonic acid (high $\mathrm{pCO}_{2}$ ) with favorable climatic conditions during weathering. However, this assertion requires further investigation, especially by dating supergene minerals formed under weathering processes/periods in order to integrate them in the local/global geological context [70,71]. 
Table 3. Supergene malachite and azurite occurrences in some Cu-deposits in Morocco and around the world.

\begin{tabular}{|c|c|c|c|c|}
\hline Deposits & Localisation & Host Rocks & Mineralization & References \\
\hline Jbel N'Zourk & $\begin{array}{l}\text { Bou Azzer-El Graara and } \\
\text { Zenaga inliers (Morocco) }\end{array}$ & Basal Series-Tamjout Dolomite & $\begin{array}{c}\text { Cu-oxides, malachite and rare traces } \\
\text { of azurite. Primary sulfides rarely } \\
\text { observed, similar as Tizert }\end{array}$ & $\begin{array}{l}\text { Clavel \& Leblanc (1971); } \\
\text { Maacha et al. (2011) [62,63] }\end{array}$ \\
\hline Tazalaght & Aït Abdallah inlier (Morocco) & $\begin{array}{l}\text { Quartzite "XII" to Lower } \\
\text { Limestone }\end{array}$ & Cu-sulfides, malachite and azurite & $\begin{array}{l}\text { Asladay et al. (1998); El } \\
\text { Basbas et al. (2011) [5,67] }\end{array}$ \\
\hline Jbel La'Sal & $\begin{array}{l}\text { Bou Azzer-El Graara inliers } \\
\text { (Morocco) }\end{array}$ & Lower part of Cambrian cover & $\begin{array}{l}\text { Malachite, chalcocite, native } \mathrm{Cu} \text {, } \\
\text { chrysocole and cuprite }\end{array}$ & Maacha et al. (2011) [64] \\
\hline Ouansimi & $\begin{array}{l}\text { Ouansimi-Kerdous inliers } \\
\text { (Morocco) }\end{array}$ & Basal Series-Lower Limestone & $\begin{array}{l}\text { Pyrite, chalcopyrite, bornite, covellite, } \\
\text { cuprite, chalcocite, malachite, azurite } \\
\text { and native } \mathrm{Cu}\end{array}$ & $\begin{array}{c}\text { Annich (2002); El Basbas et al. } \\
(2020)[7,8]\end{array}$ \\
\hline Agoujgal & Kerdous inlier (Morocco) & $\begin{array}{c}\text { Basal Series-Tamjout } \\
\text { Dolomite-Agoujgal Limestone }\end{array}$ & $\begin{array}{c}\text { Chalcopyrite, pyrite bornite, } \\
\text { chalcocite, covellite, malachite, } \\
\text { azurite, galena, sphalerite, Fe-oxides }\end{array}$ & $\begin{array}{l}\text { Asladay et al. (1998); } \\
\text { Maddi et al. (2011) [5,9] }\end{array}$ \\
\hline Luiswishi & High-Katanga (DRC) & $\begin{array}{l}\text { Neoproterozoic siliciclastic and } \\
\text { carbonate sedimentary rock }\end{array}$ & $\begin{array}{l}\text { Chalcopyrite, carrollite, pyrite, } \\
\text { malachite, heterogenite, bornite, } \\
\text { chalcocite and goethite }\end{array}$ & Fontaine et al. (2020) [66] \\
\hline Yangla & Saniang Region (China) & Metamorphic rocks & $\begin{array}{c}\text { Pyrite, chalcopyrite, pyrrhotite, } \\
\text { molybdenite, galena, sphalerite, } \\
\text { azurite, malachite }\end{array}$ & Du et al. (2020) [68] \\
\hline Tsumeb & Northern Namibia & $\begin{array}{l}\text { Otavi Group: limestone and } \\
\text { dolomite }\end{array}$ & $\begin{array}{c}\text { Malachite, azurite, wulfenite, } \\
\text { dioptase, galena, tennantite, pyrite, } \\
\text { chalcopyrite, digenite, djurleite,... }\end{array}$ & Miller (2008) [69] \\
\hline
\end{tabular}


The Tizert area experienced several geodynamic events during its geological history. The last major uplift, which probably led to weathering of the deposit, was initiated around $30 \mathrm{Ma}$, with an average erosion rate of $0.08 \mathrm{~km} / \mathrm{Ma}$ [30]. Consequently, the Meso-Cenozoic cover of this area has mostly been eroded and the Lower Cambrian terrains probably remained under a cover of sediments until the Neogene [26], prior to their exposure to surface/weathering conditions. The lowermost Cambrian is mainly characterized by Lower Limestone series, with notably, the Tamjout Dolomite (Figures 3 and 4 ) which displays a relatively high porosity, and thus, allows an efficient permeability, where supergene fluids are able to percolate. Moreover, the Basal Series were deposited in an extensional context, in basins delimited by normal faults that are not observed in the Tamjout Dolomite and overlying limestones [4,72]. Such fault systems also facilitate the percolation of supergene fluids within the Basal Series and have an impact on the depth of weathering. In addition, the occurrence of primary mineralization is generally related to the varying thickness of the Basal Series (0 to $100 \mathrm{~m}$ ) associated with paleohighs [4].

\subsection{Chemical Migration during Weathering}

Most of the primary and secondary mineralizations are concentrated in the greenish siltstone of the Basal Series: Cu-enrichment is the highest, followed by $\mathrm{Ag}$ and $\mathrm{Pb}$ (up to 100 times compare to fresh rocks). Moreover, no major depletion in metals has been identified in weathered rocks.

Other elements are slightly enriched in the weathered ore, such as As, which is generally higher in malachite-rich samples. Such high As contents are generally associated to $\mathrm{Pb}, \mathrm{Zn}$ and Ag enrichments. However, the source of As is not clearly identified. As could originate from fresh rocks, since primary sulfides, such as pyrite, galena and chalcopyrite, are devoid of this metal. Moreover, the presence of arsenopyrite (not observed) is not excluded. The authors in reference [4] determined an average grade of $23 \mathrm{~g} / \mathrm{t}$ of $\mathrm{Ag}$ in the deposit. However, only one sample displays an Ag-mineral (stromeyerite, TIZ C75 C7). By taking an average of weathered samples (oxides, secondary sulfides, etc.), the Ag content reaches $\sim 13 \mathrm{ppm}$ (from 0.5 to $62.5 \mathrm{ppm}$ ). No correlation could be made between the Ag content, the nature of the rock and the mineral phases. In the Tizert deposit, galena shows low Ag content ( $<5$ wt.\%). However, high Ag concentrations in galena have been identified in many deposits: in Morocco, Imiter deposit (Anti-Atlas) [73,74] and Tighza polymetallic district (Hercynian Belt, Central Morocco) [75], and elsewhere around the world, for example, the Broken Hill deposits [76]. At Tizert, the absence of chlorargyrite which was observed by the authors in reference [4] could be explained by its very local formation due to anthropogenic influences [77,78], even if chlorargyrite also forms naturally under arid and tropical climates e.g., references [79-81]. Native silver is stable under reduced conditions, see in reference [82] and is, therefore, rarely observed in the weathering zone.

Enrichment in $\mathrm{V}, \mathrm{Cu}, \mathrm{Zn}, \mathrm{As}, \mathrm{Mo}, \mathrm{Sb}, \mathrm{U}, \mathrm{Pb}$ and $\mathrm{Bi}$ is related to Fe-oxyhydroxide (e.g., TIZ 24 and TIZ 33, Figure 6C). In general, Fe-oxides concentrate immobile elements, such as U and REE [45,83]. The highest U content of Tizert's Fe-oxyhydroxides, generally associated with a Th depletion, is a typical supergene trend and is often related to the mobilization of $U$ and Th during weathering processes. Mobile and soluble $U$ in supergene fluids is preferentially leached and accumulated in neoformed oxyhydroxide minerals, while Th remains less sensitive to weathering phases or is rapidly assimilated by the first precipitating mineral phases $[84,85]$. However, these Fe-oxyhydroxides concentrated in the Tamjout Dolomite are depleted in REE compared to the UCC. Finally, the main chalcophile elements $(\mathrm{Pb}, \mathrm{Zn}, \mathrm{Cu})$ and the elements generally associated with $\mathrm{Au}(\mathrm{Sb}, \mathrm{As})$ are enriched.

\section{Conclusions}

At Tizert, the hypogene mineralization consisting of pyrite, chalcopyrite, chalcocite I, bornite I and galena, was affected by weathering processes leading to the formation of a secondary, supergene mineralization, mainly represented by malachite, covellite, bornite II and chalcocite II. The supergene mineralization is mainly hosted (in percentage and volume) in weathered greenish siltstones of the Basal Series, with a significant $\mathrm{Cu}$ enrichment compared to the fresh rocks. In addition, high $\mathrm{Cu}$ content 
can also be observed in the greyish-red conglomerates and sandstones of the Basal Series, as well as in the Tamjout Dolomite Formation, to a lower extent.

The Tizert giant copper deposit is mainly represented by the "green oxide zone", hosting significant amounts of malachite. This abundance of $\mathrm{Cu}$ carbonates is explained by the rapid neutralization of acidic supergene fluids by host rocks mainly composed of dolostones ("Lower Limestone"-Tamjout Dolomite Formation). In addition, chlorites of the Basal Series sandstones also played a role in this neutralization and resulted in the neoformation of smectites and/or kaolinite in weathered rocks. The absence of azurite at Tizert, which is quite common in supergene deposits, is due to low $\mathrm{CO}_{2}$ partial pressure and high $\mathrm{pH}$ conditions. Laterite s.s. is rarely observed in this deposit and iron oxyhydroxides are only observed in few spots ("patchwork" laterite).

Besides $\mathrm{Cu}$, only $\mathrm{Ag}$ is enriched during weathering processes, when slight enrichments in As, Zn, $\mathrm{Ba}$ or $\mathrm{Pb}$ are observed. The mineralization is relatively disseminated and homogeneous in the entire deposit, which could be a major advantage for potential mining.

Author Contributions: J.P., M.V., A.D., A.O., A.E.B., L.M. and J.Y. took part to the field campaigns. J.P. performed the experiments, analyzed and discussed the results, and wrote the paper. M.V., A.D. and J.Y. took part to the discussion. J.Y. coordinated the study. All authors have read and agreed to the published version of the manuscript.

Funding: This research received no external funding

Acknowledgments: Many thanks are due to Gaëtan Rochez (UNamur) for performing XRD analyses. We also thank N. Tumanov and J. Wouters from the PC2 platform (UNamur) for recording the XRD spectra. This research used resources of the Electron Microscopy Service, which is a member of the "Plateforme Technologique Morphologie-Imagerie" of the University of Namur (UNamur). Many thanks are due to the Managem Group for successful access to outcroups and boreholes, and to their exploration team for their help in selecting the sampling sites and in collecting field data. Michèle Verhaert thanks the Belgian Fund For Scientific Research (FNRS), for providing a FRIA PhD grant. We also express our gratitude to the reviewers for their constructive comments that significantly improved the manuscript.

Conflicts of Interest: The authors declare no conflict of interest. Some of the authors are affiliated with the Managem Group which is a Moroccan mining company.

\section{References}

1. Reich, M.; Vasconcelos, P.M. Geological and economic significance of supergene metal deposits. Elements 2015, 11, 305-310. [CrossRef]

2. Boni, M.; Mondillo, M. The "Calamines" and the "Others": The great family of supergene nonsulfide zinc ore. Ore Geol. Rev. 2015, 67, 208-233. [CrossRef]

3. Choulet, F.; Charles, N.; Barbanson, L.; Branquet, Y.; Sizaret, S.; Aomar, E.; Badra, L.; Chen, Y. Non-sulfide zinc deposits of the Moroccan High Atlas: Multi-scale characterization. Ore Geol. Rev. 2014, 56, 115-140. [CrossRef]

4. Oummouch, A.; Essaifi, A.; Zayane, R.; Maddi, O.; Zouhair, M.; Maacha, L. Geology and Metallogenesis of the Sediment-Hosted Cu-Ag Deposit of Tizert (Igherm Inlier, Anti-Atlas Copperbelt, Morocco). Geofluids 2017, 2017, 7508484. [CrossRef]

5. Asladay, A.; Barodi, E.B.; Maacha, L.; Zinbi, Y. Les minéralisations cuprifères du Maroc. Chron. Rech. Min. 1998, 531-532, 29-44.

6. Verhaert, M.; Madi, A.; El Basbas, A.; Elharkaty, M.; Oummouch, A.; Oumohou, L.; Malfliet, A.; Maacha, L.; Yans, J. Genesis of an As-Pb-rich supergene mineralization: The Tazalaght and Agoujgal Cu deposits (Moroccan Anti-Atlas Copperbelt). Econ. Geol.. in press.

7. Annich, M. Gisement de Ouansimi. In Méthode et Technique d'exploration Minière et Principaux Gisements au Maroc; El Barodi, E., Wat Anab, A., Mouttaqi, A., Annich, M., Eds.; Projet JICA/BRPM, BRPM, 2002; pp. 219-223.

8. $\quad$ El Basbas, A.; Aissa, M.; Ouguir, H.; My Lahsen, M.; Madi, A.; Zouhair, M. Ouansimi copper mineralization (Western Anti-Atlas, Morocco): Paragenetic sequence and circulation of gangue hosted paleofluids. J. Afr. Earth Sci. 2020, 162, 103692. [CrossRef] 
9. Maddi, O.; Baoutoul, H.; Maacha, L.; Ennaciri, O.; Soulaimani, A. La mine d'Agjgal au sud du Kerdou; considérations sur les gîtes stratoïdes de cuivre et argent de l'Anti-Atlas occidental et central. In Nouveaux Guides Géologiques et Miniers du Maroc, Principales mines du Maroc; Mouttaqi, A., Rjimati, E.C., Maacha, L., Michard, A., Soulaimani, A., Ibouh, H., Eds.; Notes et Mémoires du Service Géologique du Maroc; Ministère de l'Energie, des Mines, de l'Eau et l'Environnement: Rabat, Morocco, 2011; Volume 9, pp. 151-156.

10. Gasquet, D.; Ennih, N.; Liégeois, J.P.; Soulaimani, A.; Michard, A. The Pan-African Belt. In Continental Evolution: The Geology of Morocco; Michard, A., Saddiqi, O., Chalouan, A., Frizon de Lamotte, D., Eds.; Springer: Berlin/Heidelberg, Germany, 2008; pp. 33-64.

11. Michard, A.; Saddiqi, O.; Missenard, Y.; Oukassou, M.; Barbarand, J. Les grandes régions géologiques du Maroc; diversité et soulèvement d'ensemble. Géologues 2017, 197, 4-12.

12. Youbi, N.; Kouyaté, D.; Söderlund, U.; Ernst, R.E.; Soulaimani, A.; Hafid, A.; Ikenne, M.; El Bahat, A.; Bertrand, H.; Rkha Chaham, K.; et al. The 1750Ma Magmatic Event of the West African Craton (Anti-Atlas, Morocco). Precambrian Res. 2017, 236, 106-123. [CrossRef]

13. Gasquet, D.; Levresse, G.; Cheilletz, A.; Azizi-Samir, M.R.; Mouttaqi, A. Contribution to a geodynamic reconstruction of the Anti-Atlas (Morocco) during Pan-African times with the emphasis on inversion tectonics and metallogenic activity at the Precambrian-Cambrian transition. Precambrian Res. 2005, 140, 157-182. [CrossRef]

14. Sehrt, M.; Glasmacher, U.A.; Stockli, D.F.; Jabour, H.; Kluth, O. The southern Moroccan passive continental margin: An example of differentiated long-term landscape evolution in Gondwana. Gondwana Res. 2018, 53, 129-144. [CrossRef]

15. Benssaou, M.; Hamoumi, N. Le graben de l'Anti-Atlas occidental (Maroc): Contrôle tectonique de la paléogéographie et des séquences au Cambrien inférieur. Comptes Rendus Geosci. 2003, 335, 297-305. [CrossRef]

16. Soulaimani, A.; Michard, A.; Ouanaimi, H.; Baidder, L.; Raddi, Y.; Saddiqi, O.; Rjimati, E.C. Late Ediacaran-Cambrian structures and their reactivation during the Variscan and Alpine cycles in the Anti-Atlas (Morocco). J. Afr. Earth Sci. 2014, 98, 94-112. [CrossRef]

17. Burkhard, M.; Caritg, S.; Helg, U.; Robert-Charrue, C.; Soulaimani, A. Tectonics of the Anti-Atlas of Morocco. Comptes Rendus Geosci. 2006, 338, 11-24. [CrossRef]

18. Helg, U.; Burkhard, M.; Caritg, S.; Robert-Charrue, C. Folding and inversion tectonics in the Anti-Atlas of Morocco. Tectonics 2004, 23, 1-17. [CrossRef]

19. Michard, A.; Hoepffner, C.; Soulaimani, A.; Baidder, L. The Variscan belt. In Continental Evolution: The Geology of Morocco; Michard, A., Saddiqi, O., Chalouan, A., Frizon de Lamotte, D., Eds.; Springer: Berlin/Heidelberg, Germany, 2008; pp. 65-132.

20. Michard, A.; Soulaimani, A.; Hoepffner, C.; Ouanaimi, H.; Baidder, L.; Rjimati, E.C.; Saddiqi, O. The South-Western Branch of the Variscan Belt: Evidence from Morocco. Tectonophysics 2010, 492, 1-24. [CrossRef]

21. El Arabi, E.H.; Diez, J.B.; Broutin, J.; Essamoud, R. First palynological characterization of the Middle Triassic; implications for the first Tethysian rifting phase in Morocco. Comptes Rendus Geosci. 2006, 338, 641-649. [CrossRef]

22. Knight, K.B.; Nomade, S.; Renne, P.R.; Marzoli, A.; Bertrand, H.; Youbi, N. The Central Atlantic Magmatic Province at the Triassic-Jurassic boundary: Paleomagnetic and 40Ar/39Ar evidence from Morocco for brief, episodic volcanism. Earth Planet. Sc. Lett. 2004, 228, 143-160. [CrossRef]

23. Gouiza, M.; Charton, R.; Bertotti, G.; Andriessen, P.; Storms, J.E.A. Post-Variscan evolution of the Anti-Atlas belt of Morocco constrained from low-temperature geochronology. Int. J. Earth Sci. 2017, 106, 593-616. [CrossRef]

24. Oukassou, M.; Saddiqi, O.; Barbarand, J.; Sebti, S.; Baidder, L.; Michard, A. Post-Variscan exhumation of the central Anti-Altas (Morocco) constrained by zircon and apatite fission-track thermochronology. Terra Nova 2013, 25, 151-159. [CrossRef]

25. Frizon de Lamotte, D.; Zizi, M.; Missenard, Y.; Hafid, M.; El Azzouzi, M.; Maury, R.C.; Charrière, A.; Taki, Z.; Benammi, M.; Michard, A. The Atlas system. In Continental Evolution: The Geology of Morocco; Michard, A., Saddiqi, O., Chalouan, A., Frizon de Lamotte, D., Eds.; Springer: Berlin/Heidelberg, Germany, 2008; pp. 133-202. 
26. Leprêtre, R.; Missenard, Y.; Barbarand, J.; Gautheron, C.; Saddiqi, O.; Pinna-Jamme, R. Postrift history of the eastern central Atlantic passive margin: Insights from the Saharan region of South Morocco: Postrift Evolution Moroccan Margin. J. Geophys. Res. Solid Earth 2015, 120, 4645-4666. [CrossRef]

27. Frizon de Lamotte, D.; Leturmy, P.; Missenard, Y.; Khomsi, S.; Ruiz, G.; Saddiqi, O.; Guillocheau, F.; Michard, A. Mesozoic and Cenozoic vertical movements in the Atlas system (Algeria, Morocco, Tunisia): An overview. Tectonophysics 2009, 475,9-28. [CrossRef]

28. Missenard, Y.; Zeyen, H.; Frizon de Lamotte, D.; Leturmy, P.; Petit, C.; Sébrier, M.; Saddiqi, O. Crustal versus asthenospheric origin of relief of the Atlas Mountains of Morocco. J. Geophys. Res. Solid Earth 2006, 111, B03401. [CrossRef]

29. Frizon de Lamotte, D.; Saint Bezar, B.; Bracène, R.; Mercier, E. The two main steps of the Atlas building and geodynamics of the western Mediterranean. Tectonics 2000, 19, 740-761. [CrossRef]

30. Ruiz, G.M.H.; Sebti, S.; Negro, F.; Saddiqi, O.; Frizon De Lamotte, D.; Stockli, D.; Foeken, J.; Stuart, F.; Barbarand, J.; Schaer, J.P. From central Atlantic continental rift to Neogene uplift-western Anti-Atlas (Morocco). Terra Nova 2011, 23, 35-41. [CrossRef]

31. Soulaimani, A.; Hefferan, K. Le Précambrien à la bordure nord du craton ouest-africain (Anti-Atlas et Haut Atlas, Maroc) Données générales. Géologues 2017, 194, 33-36.

32. Hefferan, K.; Soulaimani, A.; Samson, S.D.; Admou, H.; Inglis, J.; Saquaque, A.; Latifa, C.; Heywood, N. A reconsideration of Pan African orogenic cycle in the Anti-Atlas Mountains, Morocco. J. Afr. Earth Sci. 2014, 98, 34-46. [CrossRef]

33. Boudzoumou, F.; Vandamme, D.; Affaton, P.; Gattacceca, J.; Ouazzani, H.; Badra, L.; Mahjoubi, E. Evidence of a Permian remagnetization in the Neoproterozoic-Cambrian Adoudounian Formation (Anti-Atlas, Morocco). Bull. l'Institut Sci. Sect. Sci. Terre 2012, 34, 15-28.

34. Aït Malek, H.; Gasquet, D.; Bertrand, J.M.; Leterrier, J. Géochronologie U-Pb sur zircon de granitoïdes éburnéens et panafricains dans les boutonnières protérozoïques d'Igherm, du Kerdous et du Bas Draa (Anti-Atlas occidental, Maroc). Earth Planet. Sci. Lett. 1998, 327, 819-826.

35. Hefferan, K.P.; Karson, J.A.; Saquaque, A. Proterozoic collisional basins in a Pan-African suture zone, Anti-Atlas Mountains, Morocco. Precambrian Res. 1992, 54, 295-319. [CrossRef]

36. Thomas, R.J.; Fekkak, A.; Ennih, N.; Errami, E.; Loughlin, S.C.; Gresse, P.G.; Chevallier, L.P.; Liégeois, J.P. A new lithostratigraphic framework for the Anti-Atlas Orogen, Morocco. J. Afr. Earth Sci. 2004, 39, 217-226. [CrossRef]

37. Thomas, R.J.; Chevallier, L.P.; Gresse, P.G.; Harmer, R.E.; Eglington, B.M.; Armstrong, R.A.; de Beer, C.H.; Martini, J.E.J.; de Kock, J.S.; Macey, P.H.; et al. Precambrian evolution of the Sirwa Window, Anti-Atlas Orogen, Morocco. Precambrian Res. 2002, 118, 1-57. [CrossRef]

38. Walsh, G.J.; Aleinikoff, J.N.; Benziane, F.; Yazidi, A.; Armstrong, T.R. U-Pb zircon geochronology of the Paleoproterozoic Tagragra de Tata inlier and its Neoproterozoic cover, western Anti-Atlas, Morocco. Precambrian Res. 2002, 117, 1-20. [CrossRef]

39. Maloof, A.C.; Ramezani, J.; Bowring, S.A.; Fike, D.A.; Porter, S.M.; Mazouad, M. Constraints on early Cambrian carbon cycling from the duration of the Nemakit-Daldynian-Tommotian boundary $813 \mathrm{C}$ shift, Morocco. Geology 2010, 38, 623-626. [CrossRef]

40. Maloof, A.C.; Schrag, D.P.; Crowley, J.L.; Bowring, S.A. An expanded record of Early Cambrian carbon cycling from the Anti-Atlas Margin, Morocco. Can. J. Earth Sci. 2005, 42, 2195-2216. [CrossRef]

41. Benssaou, M.; Hamoumi, N. L'Anti-Atlas occidental du Maroc: Étude sédimentologique et reconstitutions paléogéographiques au Cambrien inférieur. J. Afr. Earth Sci. 2001, 32, 351-372. [CrossRef]

42. Soulaimani, A.; Bouabdelli, M.; Piqué, A. The Upper Neoproterozoic-Lower Cambrian continental extension in the Anti-Atlas (Morocco). Bull. Soc. Geol. Fr. 2003, 174, 83-92. [CrossRef]

43. Holtzapffel, T. Les minéraux argileux. Préparation. Analyse diffractométrique et détermination. Soc. Géol. Nord 1985, 1, 113-117. (In French)

44. Taylor, S.R.; McLennan, S.M. The Continental Crust: Its Composition and Evolution; Blackwell Scientific Publications: Oxford, UK, 1985; p. 327.

45. Verhaert, M.; Bernard, A.; Dekoninck, A.; Lafforgue, L.; Saddiqi, O.; Yans, J. Mineralogical and geochemical characterization of supergene $\mathrm{Cu}-\mathrm{Pb}-\mathrm{Zn}-\mathrm{V}$ ores in the Oriental High Atlas, Morocco. Miner. Deposita 2017, 52, 1049-1068. [CrossRef] 
46. Sillitoe, R.H. Supergene oxidation of epithermal gold-silver mineralization in the Deseado massif, Patagonia, Argentina: Response to subduction of the Chile Ridge. Miner. Deposita 2019, 54, 381-394. [CrossRef]

47. Borg, G. The role of fault structures and deep oxidation in supergene base metal deposits Society of Economic Geologists: Littleton, CO, USA. In Supergene Environments, Processes and Products; Titley, S.R., Ed.; Society of Economic Geologists, Inc.7811 Shaffer ParkwayLittleton, CO 80127, Special Publication; Society of Economic Geologists, Inc: Littleton, CO, USA, 2009; Volume 14, pp. 121-132.

48. Ciantia, M.O.; Castellanza, R. Modelling weathering effects on the mechanical behaviour of rocks. Eur. J. Environ. Civ. Eng. 2016, 20, 1054-1082. [CrossRef]

49. Widdowson, M. Laterite and Ferricrete. In Geochemical Sediments and Landscapes; Nash, D.J., McLaren, S.J., Eds.; Blackwell Publishing Ltd.: Oxford, UK, 2008; pp. 45-94.

50. De Putter, T.; Mees, F.; Decrée, S.; Dewaele, S. Malachite, an indicator of major Pliocene Cu remobilization in a karstic environment (Katanga, Democratic Republic of Congo). Ore Geol. Rev. 2010, 38, 90-100. [CrossRef]

51. Robb, L.J. Introduction to Ore-Forming Processes; Blackwell Science Ltd.: Oxford, UK, 2005; p. 373.

52. Van Langendonck, S.; Muchez, P.; Dewaele, S.; Kaputo Kalubi, A.; Cailteux, J. Petrographic and mineralogical study of the sediment-hosted $\mathrm{Cu}-\mathrm{Co}$ ore deposit at Kambove West in the central part of the Katanga Copperbelt (DRC). Geol. Belg. 2013, 16, 91-104.

53. Guilbert, J.M.; Park, C.F., Jr. The Geology of Ore Deposits; W.H. Freeman \& Co.: New York, NY, USA, 1986; p. 983.

54. Brookins, D.G. Eh-pH Diagrams for Geochemistry; Springer: Berlin/Heidelberg, Germany, 1988; p. 176.

55. Chávez, W.X. Supergene Oxidation of Copper Deposits: Zoning and Distribution of Copper Oxide Minerals. Soc. Econ. Geol. 2000, 41, 11-21.

56. Hanor, J.S. Barite-celestine geochemistry and environments of formation. Rev. Mineral. Geochem. 2000, 40, 193-275. [CrossRef]

57. Domènech, C.; De Pablo, J.; Ayora, C. Oxidative dissolution of pyritic sludge from the Aznalcóllar mine (SW Spain). Chem. Geol. 2002, 190, 339-353. [CrossRef]

58. Arrobas, D.L.P.; Hund, K.L.; Mccormick, M.S.; Ningthoujam, J.; Drexhage, J.R. The Growing Role of Minerals and Metals for a Low Carbon Future (English); World Bank Group: Washington, DC, USA, 2017.

59. Descostes, M.; Beaucaire, C.; Mercier, F.; Savoye, S.; Sow, J.; Zuddas, P. Effect of carbonate ions on pyrite (FeS2) dissolution. Bull. Soc. Geol. Fr. 2002, 173, 265-270. [CrossRef]

60. Brown, E.H. The greenschist facies in part of Eastern Otago, New Zealand. Contrib. Mineral. Petrol. 1967, 14, 259-292. [CrossRef]

61. Vink, B.W. Stability relations of malachite and azurite. Mineral. Mag. 1986, 50, 41-47. [CrossRef]

62. Clavel, M.; Leblanc, M. Liaison entre tectonique et mineralisation cuprifere dans les dolomies infracambriennes de la region du Jbel N'Zourk (Anti-Atlas central, Maroc). Notes Serv. Geol. Maroc 1971, 237, 229-232.

63. Maacha, L.; Ennaciri, O.; Saquaque, A.; Soulaimani, A. Un gîte prometteur: Le cuivre du Jbel N'Zourk (Anti-Atlas central). In Nouveaux Guides Géologiques et Miniers du Maroc, Volume 9, Les Principales Mines du Maroc; Mouttaqi, A., Rjimati, E.C., Maacha, L., Michard, A., Soulaimani, A., Ibouh, H., Eds.; Notes et Mémoires du Service Géologique du Maroc, Rabat, Ministère de l'Energie, des Mines, de l'Eau et l'Environnement: Rabat, Morocco, 2011; Volume 564, pp. 123-127.

64. Maacha, L.; Ennaciri, O.; El Ghorfi, M.; Baoutoul, H.; Saquaque, A.; Soulaimani, A. Le cuivre oxydé du J. La'sal (boutonnière d'El Graara, Anti-Atlas central). In Nouveaux Guides Géologiques et Miniers du Maroc, Volume 9, Les Principales Mines du Maroc; Mouttaqi, A., Rjimati, E.C., Maacha, L., Michard, A., Soulaimani, A., Ibouh, H., Eds.; Notes et Mémoires du Service Géologique du Maroc, Ministère de l'Energie, des Mines, de l'Eau et l'Environnement: Rabat, Morocco, 2011; Volume 564, pp. 117-121.

65. Bourque, H.; Barbanson, L.; Sizaret, S.; Branquet, Y.; Ramboz, C.; Ennaciri, O.; El Ghorfi, M.; Badra, L. A contribution to the synsedimentary versus epigenetic origin of the $\mathrm{Cu}$ mineralizations hosted by terminal Neoproterozoic to Cambrian formations of the Bou Azzer-El Gaara inlier: New insights from the Jbel Laassel deposit (Anti-Atlas, Morocco). J. Afr. Earth Sci. 2015, 32, 723-739. [CrossRef]

66. Fontaine, L.; De Putter, T.; Bernard, A.; Decrée, S.; Cailteux, J.; Wouters, J.; Yans, J. Complex mineralogicalgeochemical sequences and weathering events in the supergene ore of the $\mathrm{Cu}-\mathrm{Co}$ Luiswishi deposit (Katanga, D.R. Congo). J. Afr. Earth Sci. 2020, 161, 103674. [CrossRef] 
67. El Basbas, A.; Aissa, M.; Ouguir, H.; Mahdoudi, M.L.; Madi, O.; Baoutoul, H.; Zouhair, M. La mine de cuivre de Tazalarht (Anti-Atlas occidental). In Nouveaux Guides Géologiques et Miniers du Maroc, Volume 9, Les Principales Mines du Maroc; Mouttaqi, A., Rjimati, E.C., Maacha, L., Michard, A., Soulaimani, A., Ibouh, H., Eds.; Notes et Mémoires du Service Géologique du Maroc, Ministère de l'Energie, des Mines, de l'Eau et l'Environnement: Rabat, Morocco, 2011; Volume 564, pp. 145-149.

68. Du, L.-J.; Li, B.; Huang, Z.-L.; Chen, J.; Zhou, J.-X.; Zou, G.-F.; Yan, Z.-F. Mineralogy, Fluid Inclusion, and Hydrogen and Oxygen Isotope Studies of the Intrusion-Related Yangla Cu Deposit in the Sanjiang Region, SW China: Implications for Metallogenesis and Deposit Type. Resour. Geol. 2020, 70, 28-49. [CrossRef]

69. Miller, R.M. The Geology of Namibia, 3 Volumes; Geological Survey of Namibia, Ministry of Mines and Energy: Windhoek, Namibia, 2008.

70. Vasconcelos, P.M. K-Ar and 40Ar/39Ar geochronology of weathering processes. Annu. Rev. Earth Planet. Sci. 1999, 27, 183-229. [CrossRef]

71. Lippolt, H.J.; Brander, T.; Mankopf, N.R. An attempt to determine formation ages of goethites and limonites by (U + Th)-4He dating. Neues Jahrb. Mineral. Monatshefte 1998, 11, 505-528.

72. Sellet, D.; Broughton, D.; Scott, R.; Hitzman, M.; Bull, S.; Large, R.; McGoldrick, P.; Croaker, M.; Pollington, N.; Barra, F. A New Look at the Geology of the Zambian Copperbelt. Econ. Geol. 2005, 100, 965-1000.

73. Pasava, J. Geochemistry and the role of anoxic sediments in the origin of the Imiter silver deposit in Morocco. Vestn. Ceskeho Geol. Ust. 1994, 69, 1-11.

74. Borisenko, A.S.; Borovikov, A.A.; Pavlova, G.G.; Kalinin, Y.A.; Nevolko, P.A.; Gushchina, L.V.; Lebedev, V.I.; Maacha, L.; Kostin, A.V. Formation conditions of Hg-silver deposition at the Imiter deposit (Anti-Atlas, Morocco). In Proceedings of the 12th Biennial SGA Meeting, Uppsala, Sweden, 12-15 August 2013; Volume 3.

75. Marcoux, E.; Nerci, K.; Branquet, Y.; Ramboz, C.; Ruffet, G.; Peucat, J.-J.; Stevenson, R.; Jébrak, M. Late-Hercynian intrusion-related gold deposits: An integrated model on the Tighza polymetallic district, central Morocco. J. Afr. Earth Sci. 2015, 107, 65-88.

76. Walter, S.G. Broken Hill-type deposits. J. Austr. Geol. Geophy. 1998, 17, 229-237.

77. Dill, H.G. Supergene Alteration of Ore Deposits: From Nature to Humans. Elements 2015, 11, 311-316. [CrossRef]

78. Dill, H.G.; Weber, B.; Botz, R. Metalliferous duricrusts. Neues Jahrb. Miner. Abh. 2013, 190, 123-195. [CrossRef]

79. Boyle, D.R. Iodargyrite as an indicator of arid climatic conditions and its association with gold-bearing glacial tills of the Chibougamau-Chapais Area Quebec. Can. Mineral. 1997, 35, 23-34.

80. Alfieris, D.; Voudouris, P.; Spry, P.G. Shallow submarine epithermal Pb-Zn-Cu-Au-Ag-Te mineralization on western Milos Island, Aegean Volcaninc Arc, Greece: Mineralogical, geological and geochemical constraints. Ore Geol. Rev. 2013, 53, 159-180. [CrossRef]

81. Golebiowska, B.; Pieczka, A.; Rzepa, G.; Matyszkiewicz, J.; Krajewski, M. Iodargyrite from Zalas (Cracow area, Poland) as an indicator of Oligocene-Miocene aridity in Central Europe. Palaeogeogr. Palaeoclimatol. Palaeocol. 2010, 296, 130-137. [CrossRef]

82. Keim, M.F.; Vaudrin, R.; Markl, G. Redistribution in silver during supergene oxidation of argentiferous galena: A case study from the Schwarzwald, SW Germany. Neues Jahrb. Mineral. Abh. J. Min. Geochem. 2016, 193, 295-309. [CrossRef] [PubMed]

83. De Putter, T.; Charlet, J.-M.; Quinif, Y. REE, Y and U concentration at the fluid-iron oxide interface in late Cenozoic cryptodolines from Southern Belgium. Chem. Geol. 1999, 153, 139-150. [CrossRef]

84. Verhaert, M.; Bernard, A.; Saddiqi, O.; Dekoninck, A.; Essalhi, M.; Yans, J. Mineralogy and Genesis of the Polymetallic and Polyphased Low Grade Fe-Mn-Cu Ore of Jbel Rhals Deposit (Eastern High Atlas, Morocco). Minerals 2018, 8, 23. [CrossRef]

85. Braun, J.J.; Pagel, M.; Muller, J.P.; Bilong, P.; Michard, A.; Guillet, B. Cerium anomalies in lateritic profile. Geochim. Cosmochim. Acta 1990, 54, 781-795. [CrossRef]

(C) 2020 by the authors. Licensee MDPI, Basel, Switzerland. This article is an open access article distributed under the terms and conditions of the Creative Commons Attribution (CC BY) license (http://creativecommons.org/licenses/by/4.0/). 